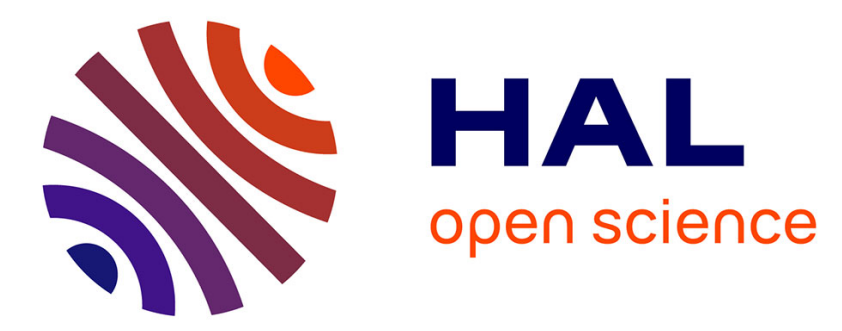

\title{
Graph models of lambda-calculus at work, and variations
} Chantal Berline

\section{To cite this version:}

Chantal Berline. Graph models of lambda-calculus at work, and variations. Mathematical Structures in Computer Science, 2006, 16, pp.185-221. 10.1017/S0960129506005123 . hal-00004473

\section{HAL Id: hal-00004473 https://hal.science/hal-00004473}

Submitted on 17 Mar 2005

HAL is a multi-disciplinary open access archive for the deposit and dissemination of scientific research documents, whether they are published or not. The documents may come from teaching and research institutions in France or abroad, or from public or private research centers.
L'archive ouverte pluridisciplinaire HAL, est destinée au dépôt et à la diffusion de documents scientifiques de niveau recherche, publiés ou non, émanant des établissements d'enseignement et de recherche français ou étrangers, des laboratoires publics ou privés. 


\title{
Graph models of $\lambda$-calculus at work, and variations
}

\author{
Chantal Berline \\ Laboratoire PPS (CNRS-Université Paris 7), \\ 2 place Jussieu (case 7014), 72251 Paris Cedex 05, France. \\ berline@pps.jussieu.fr
}

March 13, 2005

\begin{abstract}
Abstact
The paper surveys the knowledge we have acquired these last ten years about the lattice $\lambda \mathcal{T}$ of all $\lambda$-theories (= equational extensions of untyped $\lambda$-calculus), via the sets $\lambda \mathcal{C}$ consisting of the $\lambda$-theories which are representable in a uniform class $\mathcal{C}$ of $\lambda$-models. This includes positive answers to several questions raised in [9] as well as several independent results, the state of the art about the long-standing open questions concerning the representability of $\lambda_{\beta}, \lambda_{\beta \eta}$, and $H$ as theories of models, and 21 open problems.

We will focus on the class $\mathcal{G}$ of graph models since almost all the existing semantic proofs on $\lambda \mathcal{T}$ have been, or could be, more easily, obtained via graph models, or slight variations when needed. But in this paper we will also give some evidence that, for all uniform classes $\mathcal{C}, \mathcal{C}^{\prime}$ of proper $\lambda$-models living in functional semantics, $\lambda \mathcal{C}-\lambda \mathcal{C}^{\prime}$ should have cardinality $2^{\omega}$, as soon as $\mathcal{C}$ is non included in $\mathcal{C}^{\prime}$.
\end{abstract}

\section{Contents}

1 Introduction.

2 Preliminaries. 6

$2.0 .1 \quad \lambda$-toys. . . . . . . . . . . . . . . . 6

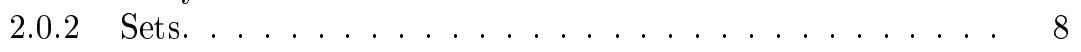

2.0 .3 Posets. . . . . . . . . . . . . . . . . . 9

2.0 .4 Lattices. . . . . . . . . . . . . . . . . . . . . 9

2.0.5 Describing the size of a poset. . . . . . . . . . . . 9

2.0.6 Scott's semantics. . . . . . . . . . . . . . . . 10

2.0.7 Other semantics of $\lambda$-calculus. . . . . . . . . . . 11 
3 The lattice of $\lambda$-theories. 11

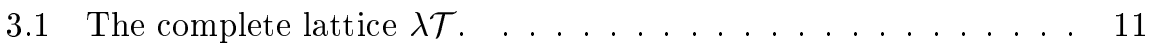

3.2 The roles of $H, B T, H^{*} \ldots \ldots \ldots \ldots \ldots \ldots \ldots$

3.3 Size and shape of the key intervals. . . . . . . . . . . . . . 13

3.4 Questioning the lattice properties of $\lambda T \ldots \ldots \ldots \ldots 14$

3.5 Representability problems. . . . . . . . . . . . . . . 15

4 Theories of ordered models. 17

4.1 Omitting $\lambda_{\beta}$ and $\lambda_{\beta \eta} \ldots \ldots \ldots \ldots \ldots \ldots \ldots \ldots \ldots$

4.2 Omitting dense sets of theories. . . . . . . . . . 18

5 Graph models. $\quad 18$

5.1 Definition. . . . . . . . . . . . . . . . . . 18

5.2 First equational and inequational properties. . . . . . . . . . . 19

5.3 Building graph models. . . . . . . . . . . . . . . . . . . 21

5.3 .1 Partial pairs. . . . . . . . . . . . . . . . 21

5.3.2 The free completion method. . . . . . . . . . . . . 21

5.3.3 The graph-product construction. . . . . . . . . . . . 23

5.3.4 The forcing completion method. . . . . . . . . . . . . 23

5.3.5 Comparing free and forcing completions. . . . . . . . . . 24

5.3.6 Generalized forcing. . . . . . . . . . . . . . . . 24

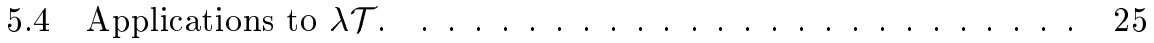

5.5 Graph models and strong surjective pairing. . . . . . . . . 26

6 The structure of $\lambda \mathcal{G}$.

6.1 Least and largest elements of $\lambda \mathcal{G}$ and $\lambda \mathcal{G}^{s} \ldots \ldots \ldots \ldots 27$

6.2 Width and height of $\lambda \mathcal{G}$ and $\lambda \mathcal{G}^{\text {den }} \ldots \ldots \ldots \ldots \ldots$

6.3 Width of $\lambda \mathcal{G}^{s} \ldots \ldots \ldots \ldots \ldots \ldots$

6.3.1 Producing 2 sensible graph theories is difficult. . . . . . 29

6.3.2 But producing $2^{\omega}$ sensible graph theories is possible. . . . 30

7 The other $\lambda \mathcal{C}^{\prime} s$.

7.1 The other key classes of models. . . . . . . . . . . . . 30

7.1.1 Comparison with $\mathcal{G}$ and $\mathcal{K}$. . . . . . . . . . . 31

7.1.2 Analogues of the key models of $\mathcal{G}$ and $\mathcal{K}$ in $\mathcal{G}_{\text {coh }}$ and $\mathcal{G}_{\text {hycoh }} .31$

7.2 Transferring the completion methods. . . . . . . . . . . 31

7.3 Transferring results from $\mathcal{G}$ to the $\mathcal{C}^{\prime} s \ldots \ldots \ldots \ldots \ldots$

7.4 Relative positions of the $\lambda \mathcal{C}^{\prime} s . \ldots \ldots \ldots \ldots \ldots$

8 Conclusion.

Keywords. Graph models, Graph semantics, untyped $\lambda$-calculus, $\lambda$-theories, models of $\lambda$-calculus, Scott's continuous semantics. 


\section{Introduction.}

In the sequel $\lambda$-calculus means Church's untyped $\lambda$-calculus, and we assume a basic knowledge of its syntax, which can be found in [4]. For this introduction $\lambda$-model only means "model of $\lambda$-calculus". In particular $\lambda$-models interpret $\lambda$ terms modulo $\alpha \beta$-conversion $\left(\lambda_{\beta}\right)$, and they are extensional if the interpretation is modulo $\alpha \beta \eta$-conversion $\left(\lambda_{\beta \eta}\right)$.

The problematic. The main concern of this paper is the study of the complete lattice $\lambda \mathcal{T}$ which consists of all $\lambda$-theories, ordered by inclusion. $\mathrm{A} \lambda$ theory is a congruence (with respect to abstraction and application) containing $\lambda_{\beta}$. Such a congruence usually arises either from syntactical and computational considerations or as the equational theory $T h(\mathcal{M})$ of a $\lambda$-model $\mathcal{M}$. The correspondence $T h$ is far from being injective, and we will also see that the question of its "surjectivity" is a key one. A $\lambda$-model $\mathcal{M}$ is called a proper model if it is not the term model of $T h(\mathcal{M})$. The best known computational theories, besides $\lambda_{\beta}$ and $\lambda_{\beta \eta}$, are the theories $H, H^{*}, B T, L T, N T$, whose definitions are recalled in Section 3.2. It is here enough to say that $B T$ equates two $\lambda$-terms iff they have the same Böhm tree, and that $L T$ and $N T$ are variants of $B T$, that $H^{*}$ and $N T$ have been proved to be equal, and finally that $B T, H$ and $H^{*}$ are sensible theories. Recall that a theory is sensible if it equates all the unsolvable terms.

We are interested in exploring the following generic questions.

Problem 1 What do models tell us about the structure of $\lambda \mathcal{T}$ ?

Given a class $\mathcal{C}$ of proper $\lambda$-models, a $\lambda$-theory $T$ is representable in $\mathcal{C}$ if there is an $\mathcal{M}$ in $\mathcal{C}$ such that $T h(\mathcal{M})=\mathcal{T}$. We denote by $\lambda \mathcal{C}$ the set of $\lambda$ theories representable in $\mathcal{C}$. The following problem is of course intimately linked to the study of $T h$.

Problem 2 What are the links between $\lambda \mathcal{T}$ and $\lambda \mathcal{C}$, where $\mathcal{C}$ is some class of proper models.

What should be very clear to the reader is that, even though $\lambda \mathcal{T}$, as well as the $\lambda \mathcal{C}^{\prime} s$ for all interesting $\mathcal{C}^{\prime} s$, all have cardinality $2^{\omega}$, only a few explicitly definable theories can be proved to be the theory of a proper $\lambda$-model. Among these exceptions are $B T, L T$ and $N T$. Whether $\lambda_{\beta}, \lambda_{\beta \eta}$ and $H$ are representable are long-standing questions, respectively due to Barendregt and Honsell, which will be discussed all along the paper.

We already surveyed in Section 6 of [9] the state of the art concerning the three generic problems presented in this introduction, and we listed a number of related open questions. In the last four years significant progress was brought in a series of papers, mainly by Antonino Salibra, whether alone or in collaboration with Bucciarelli, Lusin or the author. This makes it worthwhile to present in this paper the new state of the art on $\lambda \mathcal{T}$, reorganize the material, update the list of problems and present new ones, which we consider as very natural in view of the new picture. 
The conclusion of the paper will recapitulate the new results, and will in particular make precise the questions raised in the previous survey which have been brought an answer, following the order in which these questions appear there.

To express the results we are interested in, we found it convenient to introduce the notions of $2^{\omega}$-high, $2^{\omega}$-wide and $2^{\omega}$-broad (a notion stronger than $2^{\omega}$-wide), which will allow us to make precise three interesting possible ways for $\lambda \mathcal{T}$ and the $\lambda \mathcal{C}^{\prime} s$ of being of cardinality $2^{\omega}$.

Models and semantics. As it is well known, it took nearly 40 years to construct a proper $\lambda$-model (Scott 1969), and for this Scott had to establish Scott's continuous semantics. This first model, named $\mathcal{D}_{\infty}$, was an extensional model, built as an inverse limit. Soon afterwards, two models admitting a much simpler construction, $\mathcal{P}_{\omega}$ and $\mathcal{E}$, were proposed: respectively by Scott and Plotkin (see e.g. [4, p. 469]) and by Engeler [22] and Plokin (cf. [45]). More generally, the class $\mathcal{G}$ of graph models, which are called Engeler-Plotkin-Scott algebras in [40], was isolated $\left(\mathcal{E}\right.$ and $\left.\mathcal{P}_{\omega} \in \mathcal{G}\right)$. Let us recall that $\mathcal{E}$ is the simplest of all proper $\lambda$-models, and that all the graph models, and more generally all the models we call "webbed models" (see below) can be seen as more or less sophisticated, variants of $\mathcal{E}$. Let us recall also that $T h\left(\mathcal{P}_{\omega}\right)=T h(\mathcal{E})=B T$, and $T h\left(\mathcal{D}_{\infty}\right)=H^{*}=N T$ [27][56][40] (cf [4, Chapter 19]).

A graph model is described from a pair $(D, i)$, called its web, where $D$ is an infinite set and $i$ an injection from $D^{*} \times D$ into $D$, where $D^{*}$ is the set of all finite subsets of $D$. Such a model interprets a $\lambda$-term as a subset of $D$, and abstractions as codes in $\mathcal{P}(D)$ of Scott's continuous functions on $\mathcal{P}(D)$, the code being computed using $i$. Hence all the graph models belong to $\mathcal{C}_{\text {cont }}$, where $\mathcal{C}_{\text {cont }}$ is the class of all the $\lambda$-models which can be built inside Scott's semantics. We let $\mathcal{G}^{\text {den }}$ be the class of graph models with countable web; without loss of generality one can suppose in this case that $D=N$, nevertheless $\lambda \mathcal{G}^{\text {den }}$ is very rich.

More generally, webbed models can be defined, roughly speaking, as those $\lambda$-models which interpret $\lambda$-terms in some $\mathcal{R}(D) \subseteq \mathcal{P}(D)$, for some set $D$, and abstractions by codes in $\mathcal{R}(D)$ of functions on $\mathcal{R}(D)$ enjoying some specific properties, including continuity. Graph models form the simplest class of webbed models.

Section 5 presents graph models, the methods available for building them, and some of the equational and inequational properties which they all share. There are two known ways of building graph models: free and forcing completions of a partial web. Free completion mimics and generalizes the construction of $\mathcal{E}$. It is, essentially, a recursive procedure, similar in essence to defining intersection type systems [16], although much more general in some sense, but where redundancies are systematically cleaned. As noticed by Bucciarelli and Salibra, free completion also allows us to define a notion of product in $\mathcal{G}$ [14]. Forcing completion is a non recursive procedure, which allows one, in particular, 
to prove the easiness of $\Omega$ (i.e. the fact that $\Omega=t$ is consistent with $\lambda_{\beta}$ for all closed term $t$ ) via graph models [3]. With Salibra, we recently generalized this method and used this to prove that $\lambda \mathcal{G}$, and some related sets of $\lambda$-theories, are $2^{\omega}$-broad, and that moreover $\lambda \mathcal{T}$ contains an interval sublattice which is distributive, $2^{\omega}$-high and $2^{\omega}$-broad. Let us define a positive graph model as a model which can be presented as the free completion of a positive partial pair, in the sense of [9] or of Section 5.3.1. Positive graph models are sensible (the converse is false). In contrast, models built by forcing are most of the time non sensible.

In Section 6 we will study the structure of $\lambda \mathcal{G}$ and of $\lambda \mathcal{G}^{s}$, which is the class of theories of sensible graph models. In particular, we will see that $\lambda \mathcal{G}, \lambda \mathcal{G}^{\text {den }}$ and $\lambda \mathcal{G}^{s}$ are $2^{\omega}$-broad; whether $\lambda \mathcal{G}$ and $\lambda \mathcal{G}^{\text {den }}$ are equal is an open question. We will also see that $\lambda \mathcal{G}^{s}$ has a largest element (Bucciarelli and Salibra [15]), which is not true for $\lambda \mathcal{G}$, and this greatest element happens to be $B T$. On the other hand we will see that several results which are true for $\lambda \mathcal{G}$ still hold for $\lambda \mathcal{G}^{s}$, but that transferring the results from $\lambda \mathcal{G}$ to $\lambda \mathcal{G}^{s}$ ranges, when possible, from trivial to highly difficult (case of the uncountability of $\lambda \mathcal{G}^{s}$ ). A key question, which we already raised in [9] and which is still open, concerns the existence of a (strong) approximation theorem allowing us to manage uniformly all the positive graph models. Since we now know that $\lambda \mathcal{G}^{s}$ admits $B T$ as a top element, this would imply that $B T$ is the unique positive graph theory.

We focus on the class $\mathcal{G}$ of graph models for the following reasons. First, all the recent positive results on $\lambda \mathcal{T}$ which were proved semantically have been proved using $\mathcal{G}$, and second, most of the results ever obtained on $\lambda \mathcal{T}$ via other subclasses of $\mathcal{C}_{\text {cont }}$ could have been obtained using $\mathcal{G}$, at a significantly lower cost. Of course this has to be taken with some salt, since, for example, $\mathcal{G}$ contains no extensional models. But when dealing with extensional theories the same philosophy applies to Krivine's extension $\mathcal{K}$ of $\mathcal{G}$ (see below and in Section 7.1). In particular, $\mathcal{D}_{\infty}$ and its variants can be presented as elements of $\mathcal{K}$, which is the simplest way of getting rid of the inverse limit construction, and the simplest way of studying these models [38]. In Section 5.2 we will however give an example of equations which are consistent with $\lambda_{\beta}$ but can't be satisfied by models of $\mathcal{K}$, and in particular of $\mathcal{G}$ (Corollary 38), with the consequence that the easiness of the $\lambda$-term $\Omega_{3} I$ cannot be proved using models of $\mathcal{K}$; however Honsell and al. [1] have recently shown that this easiness can be proved semantically using filter models. Except for this example, the interest of classes wider than $\mathcal{G}$ or $\mathcal{K}$ only shows up when one needs a model whose underlying domain cannot be a complete lattice, e.g. because one wishes to model some extension of $\lambda$-calculus involving added constants with special behaviours.

Incidentally, let us recall two other classes of problems where using graph models makes life easier. First, the simplest models of $\mathcal{G}$, to begin with $\mathcal{E}$, can be used for proving some operational or syntactic properties of lambda-calculus in a more economical and more fluid way than when using intersection type 
systems (cf. [9]). Second, $\mathcal{G}$ and $\mathcal{K}$ can even prove interesting for showing the consistency of extensions of $\lambda$-calculus involving new constants; the example of the strong surjective pairing [38] is sketched in Section 5.5. To summarize, most of the time, using $\mathcal{G}$ (or $\mathcal{K}$ when necessary) brings the same information than using wider classes of models of $\mathcal{C}_{\text {cont }}$ for studying $\lambda \mathcal{T}$, more elegantly and at a significantly lower cost.

Section 7 deals with $\mathcal{K}$, and with classes $\mathcal{C}$ belonging to other semantics than Scott's one. The main variants (or refinements) of Scott's semantics are Berry and Girard's stable semantics, Bucciarelli and Ehrard's strongly stable semantics, and various weakly continuous semantics (a survey on these semantics and the diverse classes of models, as well as a complete bibliography can be found in [9]). What makes these semantics workable is the existence, in each of them, of classes analogous to $\mathcal{G}, \mathcal{K}$ (see Section 7); moreover some models of $\mathcal{C}_{\text {cont }}$, like $\mathcal{D}_{\infty}, \mathcal{E}$ and $\mathcal{P}_{\omega}$ have analogues in these classes. Hence, in general, all the positive results proved for $\lambda \mathcal{G}$ and $\lambda \mathcal{K}$ can be transferred to these other classes, in particular all are $2^{\omega}$-large and $2^{\omega}$-incomplete. Thus, the new information we get on $\lambda \mathcal{T}$ using these classes rather arises from the following third generic question:

Problem 3 What are the relative positions of the diverse $\lambda \mathcal{C}$ 's in $\lambda \mathcal{T}$.

The deepest results on Problem 3, which compare $\lambda \mathcal{C}_{\text {cont }}$ and its stable and strongly stable analogues, were obtained in the nineties and were already surveyed in [9]. The information we add here is, first, that these results still hold (and more easily) when one replaces $\mathcal{C}_{\text {cont }}$ by $\mathcal{G}$ and, second, that one can largely amplify some of these results for free by using generalized forcing instead of usual forcing, since this allows us to prove the $2^{\omega}$-broadness of some sets of the shape $\lambda \mathcal{C}-\lambda \mathcal{C}^{\prime}$, instead of their mere non-emptyness. For discussing this we also have to go deeper in the presentation of the earlier results than we did in [9]. We will also see (end of Section 2) that game semantics, although it has attracted much interest these last ten years in the context of typed $\lambda$-calculi, is not really relevant here.

Let us end this introduction by a remark which also open questions. As we will see, subsets of $\lambda \mathcal{T}$ can be proved to be $2^{\omega}$-wide or $2^{\omega}$-broad either by syntactic or by semantic means, but at present it seems we can't say anything non trivial on the height of $\lambda \mathcal{G}$, and hence on any interesting $\lambda \mathcal{C}$.

\section{Preliminaries.}

\subsection{1 $\lambda$-toys.}

$\lambda$-calculus. $\Lambda$ and $\Lambda^{\circ}$ are, respectively, the set of $\lambda$-terms and of closed $\lambda$ terms (also called combinators). Concerning specific $\lambda$-terms we set: $I \equiv \lambda x . x$, $\varepsilon \equiv \lambda x \lambda y . x y, V \equiv \lambda x \lambda y . x, F \equiv \lambda x . \lambda y . y, \Omega \equiv \delta \delta$, where $\delta \equiv \lambda x . x x$, .and 
$\Omega_{3} \equiv \delta_{3} \delta_{3}$ where $\delta_{3} \equiv \lambda x . x x x$; a more traditional notation for $V$ is $K$ (when not viewed as a boolean). We will denote $\alpha \beta$-conversion by $\lambda_{\beta}$ and $\alpha \beta \eta$-conversion by $\lambda_{\beta \eta}$. Finally we recall that the $\operatorname{order}$ of a $\lambda$-term $u$ is the largest integer $n \geq 0$ such that $u$ is $\alpha \beta$-convertible to $\lambda x_{1} \ldots \lambda x_{n} . u^{\prime}$ for some $u^{\prime}$, if there is one, and $\infty$ if this is true for all $n$.

$\lambda$-theories. A $\lambda$-theory is a congruence on $\Lambda$ (with respect to the operators of abstraction and application) which contains $\lambda_{\beta}$; it can also be seen as a (specific) set of equations between $\lambda$-terms. The set of all $\lambda$-theories will be denoted by $\lambda \mathcal{T}$. We will often drop the " $\lambda$ " of " $\lambda$-theory", since we will not use any other kind of theories here. A $\lambda$-theory is extensional if it contains the equation $I=\varepsilon$, and we let $\lambda \mathcal{T}_{e}$ be the set of extensional theories. It is clear that $\lambda_{\beta}$ and $\lambda_{\beta \eta}$ are, respectively, the least elements of $\lambda \mathcal{T}$ and $\lambda \mathcal{T}_{e}$.

The $\lambda$-theory generated (or axiomatized) by a set $E$ of equations is the least $\lambda$-theory containing it, denoted by $T_{E}$. A theory $T$ is finitely axiomatizable if $T=T_{E}$ for some finite $E$; then it is clear from the existence of a pair constructor $\lambda x \lambda y \lambda z . z x y$ in $\Lambda$, that $T=T_{E}$ for some singleton set $E$. We recall that $E \vdash r=s$ means that $r=s \in T_{E}$. A set of equations $E$ is inconsistent if $T_{E}=T o p \equiv \Lambda \times \Lambda$; in particular Top is the unique inconsistent $\lambda$-theory. For example $V=F$ is inconsistent, as well as $F=I$.

We do not ask for consistency in the definition of " $\lambda$-theory", since it will be convenient to consider Top as a $\lambda$-theory (which is obviously extensional).

Sensible $\lambda$-theories. A $\lambda$-theory is sensible if it is consistent and all the unsolvable terms are congruent, and is semi-sensible if it is consistent and no solvable term is congruent to an unsolvable term; obviously $\lambda_{\beta}$ and $\lambda_{\beta \eta}$ are semisensible and non sensible. It is well known (and easy to prove) that sensible theories are semi-sensible.

Easiness. Given a $\lambda$-theory $T$, a closed term $u$ is $T$-easy if for all other $t \in \Lambda^{0}$ we have that $T \cup\{u=t\}$ is consistent. It is easy to check that a $T$-easy term is necessarily unsolvable. On the other hand, the consistency of $T^{\prime}=T \cup\{u=I\}$, implies that $u=\lambda x . u \notin T$ (otherwise $F=I$ is in $T^{\prime}$ ); in particular, the existence of a $T$-easy term implies that $T$ is non sensible.

A term $u$ is easy if it is $\lambda_{\beta}$-easy, namely if $\{u=t\}$ is consistent for all closed term $t$. The term $\Omega$ is the best known easy term, and it enjoys further properties which make it possible to prove semantically its easiness (via graph models), using a forcing technique developed by Baeten and Boerbom [3].

$\lambda$-models. In this paper the word $\lambda$-model can very well be understood as a generic expression covering "any possible model of untyped $\lambda$-calculus". Alternatively, it can be given the precise definition of $\lambda$-model in [4], or be defined as any reflexive object of a cartesian closed category with enough points, or just be understood as "the union of all the concrete classes of models the reader has in mind". Examples of such classes will be recalled in Sections 2.0.7 
and 7.1. Each $\lambda$-model $\mathcal{M}$ induces a $\lambda$-theory, denoted here by $T h(\mathcal{M})$ or by $=_{\mathcal{M}}$. Thus: $t={ }_{\mathcal{M}} t^{\prime}$ if and only if $t=t^{\prime} \in T h(\mathcal{M})$ if and only if $t, t^{\prime}$ have the same interpretation in $\mathcal{M}$. We will call proper $\lambda$-model any $\lambda$-model $\mathcal{M}$ which is not a term model. If $\mathcal{M}$ is a proper model then $T h(\mathcal{M}) \neq T o p$, since otherwise $\mathcal{M}$ would be a singleton model and hence the term model of Top. Finally, a $\lambda$-model is sensible in case $T h(\mathcal{M})$ is.

\section{Representability of theories in classes of models.}

\section{Definition 1 Given a $\lambda$-theory $T$}

(i) $A \lambda$-model $\mathcal{M}$ is a model of $T$ if $T \subseteq T h(\mathcal{M})$.

(ii) $A \lambda$-model $\mathcal{M}$ represents $T$ if $T=T h(\mathcal{M})$.

(iii) $T$ is representable if it is representable by a proper $\lambda$-model.

Definition 2 Given a class $\mathcal{C}$ of $\lambda$-models and a theory $T$.

(i) $\mathcal{C}$ represents $T$ if there is some $\mathcal{M} \in \mathcal{C}$ representing $T$.

(ii) $\mathcal{C}$ omits $T$ if there is no $\mathcal{M} \in \mathcal{C}$ representing $T$.

(iii) $\mathcal{C}$ is complete for $\mathcal{S} \subseteq \lambda \mathcal{T}$ if $\mathcal{C}$ represents all the elements of $\mathcal{S}$.

(iv) $\mathcal{C}$ is $2^{\omega}$-incomplete if it omits $2^{\omega} \lambda$-theories.

Notation $3 \lambda \mathcal{C}$ is the set of $\lambda$-theories which are representable in $\mathcal{C}$.

$\lambda \mathcal{C}^{e}$ is the set of extensional $\lambda$-theories which are representable in $\mathcal{C}$.

$\lambda \mathcal{C}^{s}$ is the set of sensible $\lambda$-theories which are representable in $\mathcal{C}$.

$\lambda \mathcal{T}$ happens to be the instantiation of $\lambda \mathcal{C}$ where $\mathcal{C}$ consists of all possible term models, but from now on $\mathcal{C}$ will always denote a class whose definition does not refer to the syntax of $\lambda$-calculus. We will also assume that $\mathcal{C}$ is uniform, in the sense that it should not be defined as a union (definition by case). Such a class has no reason to contain term models, and in practice none does. To be more concise we will adopt the following convention.

Claim 4 From now on $\mathcal{C}$ denotes a uniform class of proper models.

In particular $\mathcal{C}$ can be any of the concrete classes of models that we will introduce later on.

\subsubsection{Sets.}

$N$ denotes the set of positive integers. For every set $S, \operatorname{card}(S)$ denotes the cardinality of $S, S^{*}$ is the set of all finite subsets of $S, \mathcal{P}(S)$ is the powerset of $S$, and $S^{<\omega}$ (resp. $S^{\omega}, S \leq \omega$ ) is the set of all finite (resp. infinite, resp. finite or infinite) sequences of elements of $S ; l(\bar{s})$ denotes the length of the sequence $\bar{s}$. When writing $g(\bar{x})$, where $g$ is a function, we will always understand that $l(\bar{x})$ is the arity of $g$. For any function $f: S \rightarrow S^{\prime}$ we define $f^{+}: \mathcal{P}(S) \rightarrow \mathcal{P}\left(S^{\prime}\right)$ by $f^{+}(A)=\{f(x): x \in A\}$ and $f^{-}: \mathcal{P}\left(S^{\prime}\right) \rightarrow \mathcal{P}(S)$ by $f^{-}(B)=\{x: f(x) \in B\}$. 


\subsubsection{Posets.}

Partially ordered sets are called posets for short. The least (or bottom) element of a poset, if any, is denoted by $\perp$. Given a set $S$, the flat partial order $S_{\perp}$ is the poset obtained from $S$, viewed as a discrete ordered set, by adding a bottom element, $\perp$. The interval notation will have the obvious meaning; for example, given a poset $\mathcal{S} \equiv(S, \sqsubseteq)$ and $s, s^{\prime} \in S$, we let $\left[s, s^{\prime}\right] \equiv\left\{s^{\prime \prime} \in S / s \sqsubseteq s^{\prime \prime} \sqsubseteq s^{\prime}\right\}$ and $\left[s, s^{\prime}\left[=\left[s, s^{\prime}\right]-\left\{s^{\prime}\right\}\right.\right.$. We say that $A \subseteq S$ is closed downwards in $\mathcal{S}$ if $l \in A$ and $l^{\prime} \sqsubseteq l$ imply $l^{\prime} \in A$ and the definition of closed upwards is symmetric.

Given a poset $\mathcal{S} \equiv(S, \sqsubseteq)$, and $S^{\prime} \subseteq S$ we recall that: $S^{\prime}$ is a a chain of $\mathcal{S}$ if it is totally ordered by $\sqsubseteq$, and $S^{\prime}$ is discrete in case its elements are pairwise incomparable for $\sqsubseteq$, which means that, for all $s, s^{\prime} \in S^{\prime}, s \sqsubseteq s^{\prime}$ implies $s=s^{\prime}$. $S^{\prime}$ is dense in $\mathcal{S}$ if $\operatorname{card}\left(S^{\prime}\right) \geq 2$ and for all distinct $s, s^{\prime} \in S^{\prime}$ we have that $] s, s^{\prime}\left[\cap S^{\prime}\right.$ is non empty, and $\mathcal{S}$ itself is a dense poset if $S$ is dense in $\mathcal{S}$. Finally, $S^{\prime}$ is an antichain of $\mathcal{S}$ if, whenever we are given $s, s^{\prime} \in S^{\prime}, s \neq s^{\prime}$, the top element $\mathrm{T}$, if there is one, is the only possible common upper bound of $s, s^{\prime}$ in $\mathcal{S}$.

\subsubsection{Lattices.}

A lattice is a poset $(S, \leq)$ such that any two elements $s, s^{\prime} \in S$ have a least upper bound $s \vee s^{\prime}$ and a greatest lower bound $s \wedge s^{\prime}$; then $\leq$ is definable from $\vee$ or $\wedge$. A lattice is complete if any $A \subseteq S$ has a least upper bound (then all $A$ have also a greatest lower bound); in particular a complete lattice has a top and a bottom element. An interval sublattice of the lattice $(S, \leq)$ is an interval which is closed under $\vee$ and $\wedge$, and, hence, is a sublattice; in particular each closed interval $\left[s, s^{\prime}\right]$ of a lattice is a lattice interval. A lattice identity is an equation $P=Q$ where $P, Q$ are terms in the language $\{\vee, \wedge\}$; a lattice identity is trivial if it holds in all lattices. A well known and useful non trivial lattice identity is distributivity: $x \wedge(y \vee z)=(x \wedge y) \vee(x \wedge z)$; a weaker condition is modularity, which expresses that distributivity holds whenever $y \leq x$.

\subsubsection{Describing the size of a poset.}

We introduce now the following definitions, which will be useful to express how large, in some various senses, some subsets of $\lambda \mathcal{T}$ can be. First we recall that a poset $\mathcal{S}=(S, \sqsubseteq)$ embeds a poset $\mathcal{S}^{\prime}=\left(S^{\prime}, \sqsubseteq^{\prime}\right)$ if there is an injection $f: S^{\prime} \rightarrow S$ such that for all $x, y \in S^{\prime}$ we have: $x \sqsubseteq^{\prime} y$ if and only if $f(x) \sqsubseteq f(y)$.

Definition 5 A poset $\mathcal{S}$ is $c$-high (resp. $c$-wide, c-broad), where $c$ is a cardinal, if $\mathcal{S}$ has a chain (resp. a discrete subset, an antichain) of cardinality $c$.

Lemma 6 If a complete lattice embeds a dense poset, then it also embeds the reals $(R,<)$ and hence it is $2^{\omega}$-high.

Lemma 7 If an ordered set embeds $(\mathcal{P}(N), \subseteq)$, then it is $2^{\omega}$-high and $2^{\omega}$-wide.

Proof. Height follows from Lemma 6, once noticed that $\left(\mathcal{P}^{\infty}(N), \subseteq \infty\right)$ is dense, where $\mathcal{P}^{\infty}(N)$ is the set of infinite subsets of $N$ and $A \subseteq \infty B$ if $A \subseteq B$ 
and $B-A$ is empty or infinite. Width comes from the fact that it is easy to build $2^{\omega}$ pairwise incomparable subsets of $N$, for example $\left\{A_{f} / f: P \rightarrow P\right\}$, where $P$ is the set of prime numbers and $A_{f} \equiv\left\{p^{f(p)} / p \in P\right\}$.

\subsubsection{Scott's semantics.}

Cpos (complete partial orders) and (Scott-) continuous functions between cpos are defined in [4, Chapter I.2]; all complete lattices are cpos. Two cpos $\mathcal{D}$ and $\mathcal{D}^{\prime}$ are Scott-isomorphic if and only if they are isomorphic as posets, namely if there is a bijection between them such that $f(x)<f(y)$ if and only if $x<y$, and we will denote this by $\mathcal{D} \simeq \mathcal{D}^{\prime}$. If $\mathcal{D}, \mathcal{D}^{\prime}$ are cpos then $\left[\mathcal{D} \rightarrow \mathcal{D}^{\prime}\right]$ denotes the cpo of all the continuous functions from $\mathcal{D}$ into $\mathcal{D}^{\prime}$. A reflexive cpo is a triple $(\mathcal{D}, A, \lambda)$ such that $\lambda \in[[\mathcal{D} \rightarrow \mathcal{D}] \rightarrow \mathcal{D}]$ and $A \in[\mathcal{D} \rightarrow[\mathcal{D} \rightarrow \mathcal{D}]]$ and $A \circ \lambda=i d$. Reflexive cpos model $\lambda$-calculus as follows (for more details see [4, Chapter V.5]).

Let $E n v_{\mathcal{D}}$ be the set of environments $\rho$ mapping the set of the variables of $\lambda$-calculus into $\mathcal{D}$. For $\rho \in E n v$ and $d \in \mathcal{D}$ let $\rho[x: d]$ be the environment which takes value $d$ on $x$ and agrees with $\rho$ on the other variables. The interpretation $|t|: \operatorname{Env}_{\mathcal{D}} \rightarrow \mathcal{D}$ of a $\lambda$-term $t$ which is relative to $(\mathcal{D}, A, \lambda)$ is defined by induction as follows: (i) $|x|_{\rho}=\rho(x)$, (ii) $|t u|_{\rho}=A\left(|t|_{\rho}\right)\left(|u|_{\rho}\right)$ and (iii) $|\lambda x . t|_{\rho}=\lambda(d \in \mathcal{D} \mapsto$ $\left.|t|_{\rho[x: d]}\right)$.

Graph models are based on cpos of the form $(\mathcal{P}(D), \subseteq)$, for some infinite set $D$; such cpos are, of course, complete lattices. If $D$ is countable then a function $g: \mathcal{P}(D) \rightarrow \mathcal{P}(D)$ is continuous if and only if it is monotone and commutes with all increasing unions.

Further conventions on sets. When dealing with a graph model based on $\mathcal{P}(D)$, greek letters $\alpha, \beta, \ldots$ will always understand elements of $D$, small Latin letters $a, b, c$ will understand elements of $D^{*}$, i.e. finite subsets of $D$, and $\bar{a}, \bar{b}, \bar{c} \ldots$ elements of $\left(D^{*}\right)^{<\omega}$. Also, $(a, \alpha)$ is the usual set-theoretical pair, and $(\bar{a}, \alpha)$ is defined by induction as follows: $(\bar{a}, \alpha)=\alpha$ if $l(\bar{a})=0$ and $(b \bar{c}, \alpha)={ }_{\text {def }}(b,(\bar{c}, \alpha))$.

Traces of continuous functions. Products of cpos are again cpos. By "a continuous function $g$ of arity $n$ on $\mathcal{P}(D)$ " we mean: $g \in\left[\mathcal{P}(D)^{n} \rightarrow \mathcal{P}(D)\right]$.

A continuous function $g$ on $\mathcal{P}(D)$, of any arity, is completely determined by its trace, which is defined by:

$$
\operatorname{tr}(g)=_{\text {def }}\{(\bar{a}, \alpha): \alpha \in g(\bar{a})\}
$$

The trace can be viewed as the relevant part of the graph $\operatorname{graph}(g)$ of $g$. Note however that, in case of arity one, $\operatorname{tr}(g) \subseteq D^{*} \times D \subseteq \mathcal{P}(D) \times D$, while $\operatorname{graph}(g) \subseteq \mathcal{P}(D) \times \mathcal{P}(D)$

Graph models owe their name to the fact that continuous functions are encoded in them via (a sufficient fragment of) their graphs, namely their traces. 


\subsubsection{Other semantics of $\lambda$-calculus.}

Besides Scott's continuous semantics, the key examples are Berry's stable semantics and the Bucciarelli-Ehrhard strongly stable semantics, which are refinements of Scott's continuous semantics capturing some aspects of the sequentiality of $\lambda$-calculus. By the "three main semantics" we will understand one of these and, for brevity, we will respectively call the $\lambda$-models living inside: continuous, stable and strongly stable $\lambda$-models. These semantics are functional, in the sense that $\lambda$-terms are interpreted by morphisms which are functions; this is also the case for a further family of semantics, namely the $\kappa$-continuous semantics, $\kappa$ any regular cardinal ( $\kappa$-continuity is a weakening of Scott's continuity). No more details on these semantics than what is stated in this subsection should be needed to read this paper, and, if necessary, [9] contains a more detailed presentation.

Notation $8 \mathcal{C}_{\text {cont }}, \mathcal{C}_{\text {st }} \mathcal{C}_{\text {sts }}$ will denote respectively the classes of continuous, stable, and strongly stable $\lambda$-models, respectively.

Similarly, $\mathcal{C}_{\kappa-\text { cont }}$ and $\mathcal{C}_{\text {games }}$ will denote the classes of models corresponding to the $\kappa$-semantics and to the game semantics, respectively.

Our main concern is that of representability problems. In this context game semantics (which is not a functional semantics) is not relevant, since it was proved by Franco [20] that $\lambda \mathcal{C}_{\text {games }}$ (at least in the case of Abramsky \& al. games), only contains the theories $B T, L T$ and $H^{*}$ (see Section 3.2 for a definition of these theories) while all the functional semantics happen to be very rich and are also able to represent these theories. Since no systematic study of $\lambda \mathcal{C}_{\kappa-\text { cont }}$ has yet been undertaken, we will nearly only deal here with the three main semantics, with a great emphasis on $\mathcal{G}$ (the other subclasses of models will only be treated in Section 7).

\section{The lattice of $\lambda$-theories.}

\subsection{The complete lattice $\lambda \mathcal{T}$.}

$\lambda \mathcal{T}$, ordered by inclusion, is naturally equipped with a structure of complete lattice, where the meet of a family of $\lambda$-theories is their intersection $\cap$, and the join, written here + , is the least equivalence relation containing their union. Since $\Lambda$ is countable $\operatorname{card}(\lambda \mathcal{T}) \leq 2^{\omega}$, and we will see soon that $\lambda \mathcal{T}$ is in fact $2^{\omega}$ high and $2^{\omega}$-broad.

Two theories $T, T^{\prime}$ are incompatible if $T \cup T^{\prime}$ is inconsistent, or equivalently if $T+T^{\prime}=T o p$. Hence an antichain of $\lambda \mathcal{T}$ is a set of theories which are pairwise incompatible.

\section{Notation 9 .}

(i) $\lambda \mathcal{T}^{\text {re }}$ denotes the set of recursively enumerable $\lambda$-theories.

(ii) $\lambda \mathcal{T}^{s}$ is the set of all sensible $\lambda$-theories.

(iii) $\lambda \mathcal{T}^{s / 2}$ is the set of all semi-sensible $\lambda$-theories. 


\section{Notation 10 .}

Notation 11 (i) $\mathcal{S}^{r e}=\mathcal{S} \cap \lambda \mathcal{T}^{r e}$.

(ii) $\mathcal{S}_{e}=\mathcal{S} \cap \lambda \mathcal{T}_{e}$ will be called the extensional analogue of $\mathcal{S} \subseteq \lambda \mathcal{T}$.

It follows immediately from their definitions that $\lambda \mathcal{T}^{s}, \lambda \mathcal{T}^{s / 2}$ and their extensional analogues, are closed under (finite or infinite) intersection, that $\lambda \mathcal{T}^{s}$, $\lambda \mathcal{T}_{e}$ and $\lambda \mathcal{T}_{e}^{s}$ are closed upwards (omitting Top in the sensible case) and that $\lambda \mathcal{T}^{s / 2}$ is closed downwards. Obviously $\lambda \mathcal{T}=\left[\lambda_{\beta}, T o p\right]$ while $\lambda \mathcal{T}_{e}=\left[\lambda_{\beta \eta}, T o p\right]$, and we will see below that $\lambda \mathcal{T}^{s}, \lambda \mathcal{T}^{s / 2}$ and their extensional analogues are also closed intervals of $\lambda \mathcal{T}$, and hence complete sublattices. Concerning recursively enumerable theories, note that they are non sensible (this can be inferred from [4, Chapter 17.1]) but that plenty of them are semi-sensible, to begin with $\lambda_{\beta}$. Of course $\lambda \mathcal{T}^{r e}$ contains all the finitely axiomatizable theories.

In the next two subsections we recall briefly the "historical" results on $\lambda \mathcal{T}$. Most of them (or their proofs) are purely syntactic, and all of them can be found in [4].

\subsection{The roles of $H, B T, H^{*}$.}

Since $\lambda \mathcal{T}^{s}$ is closed under intersections, it has a smallest element, which is the intersection of all the sensible theories, and is traditionally denoted by $H$.

The theories $H, B T, H^{*}$ were the first theories to be isolated, and they happen to play an important role with respect to the structure of $\lambda \mathcal{T}$. The theory $B T$ contains $t=t^{\prime}$ if and only if the $\lambda$-terms $t, t^{\prime}$ have the same Böhm tree; hence it is a sensible theory, which is strictly bigger than $H$, and $B T$ is non extensional; thus no theory below $B T$ can be extensional; in particular $H$ is not. The theory $H^{*}$ contains $t=t^{\prime}$ if and only if for all context $C[-]$ we have that $C[t]$ is solvable if and only if $C\left[t^{\prime}\right]$ is solvable. It follows immediately from this definition that $H^{*}$ is sensible and that every semi-sensible theory is included in $H^{*}$; hence $H^{*}$ is the unique maximal sensible (resp. semi-sensible) theory. Since $\lambda \mathcal{T}^{s}$ is closed upwards, there is no theory between $H^{*}$ and $T o p$, since $\lambda \mathcal{T}^{s / 2}$ is closed downwards we get $\lambda \mathcal{T}^{s / 2}=\left[\lambda_{\beta}, H^{*}\right]$. Finally:

$$
\begin{gathered}
\lambda_{\beta} \subsetneq H \subsetneq B T \subsetneq H^{*} \subsetneq T o p \\
] H^{*}, T o p[=\emptyset \\
\lambda \mathcal{T}^{s}=\left[H, H^{*}\right]=[H, T o p[ \\
\lambda \mathcal{T}^{s / 2}=\left[\lambda_{\beta}, H^{*}\right]
\end{gathered}
$$

while, of course:

$$
\lambda \mathcal{T}=\left[\lambda_{\beta}, T o p\right]
$$

It was proved by Hyland [27] and Wadsworth [56] that $H^{*}=T h\left(\mathcal{D}_{\infty}\right)$, where we recall that $\mathcal{D}_{\infty}$ is Scott's first model; in particular $H^{*}$ is extensional. It is interesting to note that there is another characterization of $H^{*}$, in terms of trees: $H^{*}=N T$, where $N T$ equals two terms if and only if they have the same 
Nakajima tree (cf. [4, Exercise 19.4.4]). Finally, it is worth introducing Longo's theory $L T$, which is also defined in terms of trees. $L T$ is a "lazy" version of $B T$, which equates two unsolvable terms $u$ and $v$ if and only if they have the same order. We have: $L T \subseteq B T$ and $\Omega=\lambda x . \Omega \in B T-L T$, hence $L T \in \lambda \mathcal{T}^{s / 2}-\lambda \mathcal{T}^{s}$.

The extensional case. Given a theory $T$, let $T_{\eta}$ denote the smallest extensional theory containing $T$ and $I=\varepsilon$. Since $H^{*}$ is extensional, $T_{\eta}$ is consistent for all semi-sensible theory; furthermore $H_{\eta}$ is the smallest extensional sensible theory, and $H_{\eta} \subseteq B T_{\eta} \subseteq H^{*}$. In fact these inclusions are strict, so finally, in the extensional case we get similar inclusions and equalities than above, with $\lambda_{\beta \eta}, H_{\eta}$ and $B T_{\eta}$ replacing $\lambda_{\beta}, H$ and $B T$.

\subsection{Size and shape of the key intervals.}

The following results show in particular that all the intervals mentioned in the previous subsection are as high and wide as possible. The proofs of the theorems can be found in [4, Chapters 17.1 and 16.3] and the proofs of the corollaries are immediate. The proofs of the two propositions are respectively recalled and given for allowing comparison with the semantic proofs that will be given later on.

Theorem 12 (Visser 1980) If $T \in \lambda \mathcal{T}^{r e}$ and $r=s \notin T$, then there exists $u$ such that, for all $t, T \cup\{u=t\} \nvdash r=s$.

Corollary 13 If $T$ is recursively enumerable then there is a T-easy term.

Corollary 14 If $T$ is recursively enumerable then $T$ is non sensible.

Proposition $15 \lambda \mathcal{T}^{r e}$ is a dense subset of $\lambda \mathcal{T}$, and the same holds for their extensional analogues.

Proof. Let $T, T^{\prime} \in \lambda \mathcal{T}^{\text {re }}$ be such that $T \varsubsetneqq T^{\prime}$, let $r=s \in T^{\prime}-T$ and finally let $S=T \cup\{u r=u s\}$, where $u$ is given by Theorem 12. It is clear that $T \subseteq T_{S} \subseteq T^{\prime}$, that $T_{S} \in \lambda \mathcal{T}^{r e}$. Since $T_{S} \cup\{u=I\} \vdash r=s$ we have $T_{s} \neq T$ by Theorem 12 . If $T^{\prime}=T_{S}$ then $T \cup\{u r=u s\} \vdash r=s$, but $T \cup\{u=\lambda x . I\} \vdash u r=u s$, hence $T \cup\{u=\lambda x . I\} \vdash r=s$, which contradicts Theorem 12. Thus $T \varsubsetneqq T_{S} \varsubsetneqq T^{\prime}$.

Proposition 16 If $T \in \lambda \mathcal{T}^{r e}$ then $[T, T o p]$ is $2^{\omega}$-high and $2^{\omega}$-broad.

Sketch of proof. The fact that $[T, T o p]$ is $2^{\omega}$-high follows immediately from Proposition 15 and Lemma 6 . The fact that it is $2^{\omega}$-broad follows from Theorem 12 , plus a compactness argument. Let indeed $u$ be a $T$-easy term, let $\hat{n}$ denote the $\mathrm{n}$-th Church integer. It is enough to prove that for each sequence $s=\left(t_{n}\right)_{n \in \omega}$ of $\lambda$-terms, the set $E_{s}=\cup E_{s, n}$ is consistent with $T$, where $E_{s, n}=\{u \hat{1}=$ $\left.t_{1}, \ldots, u \hat{n}=t_{n}\right\}$. Indeed, if $s$ is such that $t_{n}=t_{m}$ is consistent only if $n=m$, for example if $s$ is itself the sequence of the Church integers, then the sets $E_{\sigma s}$, where $\sigma s$ is obtained from $s$ by action of the permutation $\sigma$ of $\omega$, will generate 
pairwise inconsistent theories. Now, to prove that $E_{s}$ is consistent with $T$ it is enough, by compactness, to show that each of the $T \cup E_{s, n}$ is consistent. But, using usual tricks of $\lambda$-calculus, given the sequence $s$, there is for each $n$ a $\lambda$-term $\operatorname{cond}_{n}$ such that $\operatorname{cond}_{n} \hat{\jmath}={ }_{\beta} t_{j}$ for all $j \leq n$. Now, by Theorem 12, $u=\operatorname{cond}_{n}$ is consistent with $T$, which implies the consistency of $E_{s, n} \cup T$.

Corollary $17 \lambda \mathcal{T}$ is $2^{\omega}$-high and $2^{\omega}$-broad, and similarly with $\lambda \mathcal{T}_{e}$.

Theorem 18 (Barendregt \& al. 1980)

$(\mathcal{P}(N), \subseteq)$ can be embedded in $] H_{\eta}, B T_{\eta}[$, and also in $] H, B T[$.

Corollary $19 \lambda \mathcal{T}^{s}, \lambda \mathcal{T}^{s / 2}$ are $2^{\omega}$-high and $2^{\omega}$-wide, and the same is true for their extensional analogues.

This corollary follows from Theorem 18 and from Lemma 7, since the three "extensional" classes contain $] H_{\eta}, B T_{\eta}[$, and the three other ones $] H, B T[$.

In the sequel we will see that the $2^{\omega}$-broadness of $\lambda \mathcal{T}$ and the $2^{\omega}$-width of $\lambda \mathcal{T}^{s}$ and $\lambda \mathcal{T}^{s / 2}$ can also be given semantic proofs, via $\mathcal{G}$.

We end this section by quoting a very nice recent result of Statman, that we will not use in the sequel, but which reminds us (as the existence of easy terms) that surprising things can very well happen in $\lambda \mathcal{T}$.

Theorem 20 (Statman 2001) [54]. There is an equation e $\notin \lambda_{\beta}$ which is consistent with all consistent theory.

From Corollary 17 and Zorn's Lemma it follows that the set of maximal consistent $\lambda$-theories is "maximally large", namely $2^{\omega}$-broad; and we also know that $H^{*}$ is one of its elements. Now, Statman's Theorem is equivalent to saying that there is an equation $e \notin \lambda_{\beta}$ in the intersection of all these theories.

\subsection{Questioning the lattice properties of $\lambda T$.}

At the end of the nineties, Antonino Salibra [47] launched a research program for exploring the lattice $\lambda \mathcal{T}$ using techniques of universal algebra. The first result was obtained in [47], where the lattice of $\lambda$-theories is shown to be isomorphic to the lattice of equational theories of a suitable class of algebras. Then the first important remark is that not any lattice can be a lattice of equational theories; examples of such constraints are the Zipper condition and the ET condition (cf.[41]), which are not identities. Salibra proposed the following conjecture, and recently proved with Lusin an approximation of it, which involves bounded versions of + , and can be found in [41].

Conjecture 4 (Salibra 2000) $\lambda \mathcal{T}$ satisfies no non trivial lattice identity.

Theorem 21 (Salibra 2001) [48] $\lambda \mathcal{T}$ is not modular (and hence not distributive). 
Sketch of proof. The modularity law (see Section 2.0.4) fails for $x=H_{\eta}$, $y=H$ and $z=T$, where $T$ is generated by the equation $\Omega=I$. Since $H$ contains the equation $\Omega=\lambda x . \Omega$ we have $T+H=T o p$, hence $H_{\eta} \cap(H+T)=H_{\eta}$. On the other hand $\left(H_{\eta} \cap H\right)+\left(H_{\eta} \cap T\right)=H+\left(H_{\eta} \cap T\right)$. Obviously, $\varepsilon=I \in H_{\eta}$; on the other hand $\varepsilon=I \notin H+\left(H_{\eta} \cap T\right)$ (see [48]), hence $H+\left(H_{\eta} \cap T\right) \neq H_{\eta}$. In fact the proof works with any sensible extensional theory instead of $H_{\eta}$.

Obviously, the above theorem is no longer true for sublattices, in general. For example the interval lattices $\{T o p\}$ and $\left[H^{*}, T o p\right]$, which have respectively one and two elements, satisfy a lot of identities, including distributivity.

Problem 5 (Salibra) Are there large intervals of $\lambda \mathcal{T}$ which satisfy interesting lattice identities?

There are good reasons to be interested in intervals of the form $[T, T o p]$; the first one is that $[T, T o p]$ is isomorphic to the lattice of congruences on the term-algebra $\Lambda / T$, which is a bridge to universal algebra, and a second one is that we know from Proposition 16 that for all $T \in \mathcal{T}^{\text {re }}$, and in particular for all finitely axiomatizable $T$, the interval $[T, T o p]$ is as large as it can be (as we can express it to be), namely: $2^{\omega}$-high and $2^{\omega}$-broad.

Theorem 22 (Berline and Salibra 2004) [10] There is a finitely axiomatizable theory $T$ such that $[T, T o p]$ is distributive.

The proof, which is semantic, will be given in Section 5.4 as a direct application of generalized forcing over graph models. Moreover we will have for free that $[T, T o p] \cap \lambda \mathcal{G}$ is $2^{\omega}$-broad, and hence that $\lambda \mathcal{T}$, and also $[T, T o p]$ for this $T$, are also $2^{\omega}$-broad.

\subsection{Representability problems.}

We already know that $T h\left(\mathcal{D}_{\infty}\right)=H^{*}$ and that $B T=T h(\mathcal{E})=T h\left(\mathcal{P}_{\omega}\right)$; in particular $T h$ is non injective (where we take as informal domain of definition for $T h$ the union of all conceivable $\mathcal{C}^{\prime} s$ ). In fact, as we will see, $B T$ can even be represented by $2^{\omega}$ models of $\mathcal{G}$, and $B T$ and $H^{*}$ can also be represented in each of the other main semantics! The question of the surjectivity of $T h$ is much more difficult; Salibra's Theorem 28 below is a strong, although not yet definite, indication that the answer should be negative, and that moreover the range of $T h$ is $2^{\omega}$-incomplete.

About $\lambda_{\beta}, \lambda_{\beta \eta}$ and $H$. As mentioned in the introduction, the problems of the representability of $\lambda_{\beta}, \lambda_{\beta \eta}$ and of $H$ are respectively due to Barendregt and Honsell. Both are long-standing questions (nearly as old as the existence of Scott's continuous semantics), even though they were only first discussed in print in Honsell-Ronchi [26].

Problem 6 (Barendregt; Honsell) Are $\lambda_{\beta}, \lambda_{\beta \eta}, H$ representable? 
These questions are still open. The first partial answers only concern $\lambda_{\beta \eta}$ and date back as recently as 1995, and all the other partial results obtained so far are essentially negative. Nevertheless exploring these questions allowed us to gather interesting information about the diverse $\lambda \mathcal{C}^{\prime} s$, as we will see in the next subsection. The only positive result is the following one. Note however that the model built in its proof does not really answer Barendregt's problem in spirit, since it is "essentiality syntactic", in the sense that its construction is based on the syntax of $\lambda$-calculus.

Theorem 23 (Di Gianantonio-Honsell-Plotkin 1995) [21]

$\lambda_{\beta \eta}$ is representable by (a kind of) weakly continuous model.

Hint. Let us say that a function (between adequate cpos) is DHP-continuous if it commutes with sups of increasing sequences indexed by $\omega_{1}$, and let us call $\mathcal{C}_{D H P \text {-cont }}$ the class of $\lambda$-models associated to the corresponding semantics. Starting from the term model $\Lambda_{\beta \eta}$ of $\lambda_{\beta \eta}$, the authors build an inverse limit $\mathcal{M}$ in an adequate category such that: $\mathcal{M} \in \mathcal{C}_{D H P \text {-cont }}$ and there is a function $p: \mathcal{M} \rightarrow \Lambda_{\beta \eta}$ such that $p\left(|t|^{\mathcal{M}}\right)$ is the class of $t$ modulo $\lambda_{\beta \eta}$, for all $t \in \Lambda^{0}$. Then, obviously, $\operatorname{Th}(\mathcal{M})=\lambda_{\beta \eta}$.

About the $\lambda \mathcal{C}^{\prime} s$. The question of the representability of $\lambda_{\beta}, \lambda_{\beta \eta}$, and $H$ generated a wealth of related questions. In the following, $\mathcal{C}$ denotes any uniform class of proper models we are interested in. Of course, the three following problems are not independent.

Problem 7 Is $\mathcal{C}$ complete for $\lambda \mathcal{T}$ (or some more adequate subset of $\lambda \mathcal{T}$ )?

We will see in the next section that all known semantics are $2^{\omega}$-incomplete.

Problem 8 Are $\lambda_{\beta}, \lambda_{\beta \eta}, H$ representable in $\mathcal{C}$ ? for $H$.

We will see that, for $\mathcal{C}=\mathcal{G}$, the answer is "no" for $\lambda_{\beta}, \lambda_{\beta \eta}$, and is still open

Problem 9 Is there a least element in $\lambda \mathcal{C}$ ? if yes, does it admit another (and preferably nice) characterization?

Theorem 24 (Di Gianantonio-Honsell-Plotkin 1995) [21]

$\lambda \mathcal{C}_{\text {cont }}^{e}$ has a least element.

Hint. Using the axiom of choice, choose one continuous model $\mathcal{M}_{T}$ for each theory $T \in \lambda \mathcal{C}_{\text {cont }}^{e}$. Starting from the cartesian product $\Pi$ of the $\mathcal{M}_{T}$ it is possible to build an inverse limit $\mathcal{M}$ in an adequate category, in such a way that $\mathcal{M} \in \mathcal{C}_{\text {cont }}^{e}$ and $T h(\mathcal{M}) \subseteq T h\left(\mathcal{M}_{T}\right)$ for all $T$. The proof in [21] of this last point uses "logical relations" between $\Pi$ and $\mathcal{M}$; since logical relations do not distinguish terms having the same applicative behaviour, this proof can only work with extensional models. 
Problem 10 Compare the shape of $\lambda \mathcal{C}$ with that of $\lambda \mathcal{T}$ or of a more appropriate sublattice.

These problems already appear in [9], sometimes with a different formulation. Since then several results were obtained, that we will survey from Section 5 on. In particular the case of $\mathcal{C}=\mathcal{G}$ has been thoroughly studied; but some key questions remain open.

\section{Theories of ordered models.}

\subsection{Omitting $\lambda_{\beta}$ and $\lambda_{\beta \eta}$.}

Definition 25 Let us call p.o $\lambda$-model any $\lambda$-model $\mathcal{M}$ such that application is monotone for some non trivial partial order $\leq$ on the carrier set of $\mathcal{M}$, and p. $\mathrm{O}_{\perp} \lambda$-model any p.o $\lambda$-model having a bottom element. We will write $\leq_{\mathcal{M}}$ for the preorder induced by $\leq$ on $\Lambda^{0}$; thus $t \leq_{\mathcal{M}} u$ if and only if $|t|_{\mathcal{M}} \leq|u|_{\mathcal{M}}$.

Graph models, and more generally all the proper models that we meet in $\lambda$-calculus, are p.o $o_{\perp} \lambda$-models. Not only there are well known computational motivations for considering p.o $\lambda$-models, but it takes much energy to find $\lambda$ models which are not p.o $\lambda$-models. The first one was built by Plotkin in 1995 [46], and it is only at the same time that it could be proved that the term models of $\lambda_{\beta}$ and $\lambda_{\beta \eta}$ were not orderable. This is a corollary of the following beautiful theorem due to Peter Selinger, which also gives a partial answer to Problem 8.

Theorem 26 (Selinger 96) [52][53] Let $\mathcal{M}$ be a p.o $\lambda$-model. If $T h(\mathcal{M})=\lambda_{\beta}$ or $\lambda_{\beta \eta}$, then the order is trivial on the interpretations of closed terms.

Sketch of the proof in [52]. There is a term $A \in \Lambda^{0}$ such that $A x x x y={ }_{\lambda \beta}$ Axyyy while $A x x x y \neq_{\lambda \beta \eta}$ Axxyy, for variables $x, y$. Hence, for all closed terms $t, u$ and variables $z, s, t \leq_{\mathcal{M}} u$ implies $A(s t)(s t)(s t)(z u)=_{\mathcal{M}} A(s t)(s t)(z u)(z u)$. It is then enough to prove that $A(s t)(s t)(s t)(z u) \neq_{T} A(s t)(s t)(z u)(z u)$ if $t \neq_{T} u$, where $T=\lambda_{\beta}$ or $\lambda_{\beta \eta}$. This follows from a non trivial lemma which states that if $t \neq_{T} u$ then st and $z u$ behave like distinct variables: for all $B, C \in \Lambda^{0}$, if $B(s t)(z u)=_{T} C(s t)(z u)$ then $B x y=_{T} C x y$.

Recall that the one, and only one, example we have of an $\mathcal{M}$ satisfying the hypothesis of the above theorem is the DHP-continuous model built in [21] for proving Theorem 23, which furthermore is essentially syntactic.

Problem 11 Does the above statement hold for $H$ ?

Remark 27 It follows from the proof of Theorem 26 that, for the above term $A$, we have: $\operatorname{Th}(\mathcal{M}) \supseteq\left\{A(s t)(s t)(s t)(z u)=A(s t)(s t)(z u)(z u) / t \leq_{\mathcal{M}} u\right\}$ for all p.o. $\lambda$-model $\mathcal{M}$. 


\subsection{Omitting dense sets of theories.}

In [49] Antonino Salibra proved that (except maybe for very exotic orders) all uniform classes of ordered models are $2^{\omega}$-incomplete; the proof mixes tricky arguments from topology and universal algebra. The following consequence of this result, already covers all the semantics we have met so far, and admits a simpler proof, due to Salibra and Plotkin (see [50]), which is composed of the next two lemmas. In particular, Theorem 28 answers positively Question 2 of [9, Section 6.1, p.149 ] (cf. Section Conclusion).

Theorem 28 (Salibra 2001) The class of all p.o $\perp \lambda$-models omits $2^{\omega}$-high intervals of $\lambda \mathcal{T}$.

In the following, $(V, F)$ could be be replaced by any pair $(t, r)$ of terms such that $t=r$ is inconsistent.

Let $\Pi$ and $\Pi^{\prime}$ be the $\lambda$-theories respectively axiomatized by $\{\Omega x x=\Omega\}$ and $\{\Omega x x=\Omega, \Omega=\Omega(\Omega V F) \Omega\}$. Clearly, $\Pi \subseteq \Pi^{\prime} \subseteq H$; in particular $\Pi$ and $\Pi^{\prime}$ are consistent.

Lemma 29 The interval $\left[\Pi, \Pi^{\prime}\right]$ is $2^{\omega}$-high.

Sketch of proof. Thanks to Theorem 15, it is enough to prove that $\Pi \varsubsetneqq \Pi^{\prime}$. It is proved in [50] that $(u=v) \in \Pi \Longleftrightarrow(\Omega u v=\Omega) \in \Pi$; applying twice this result to the equation $\Omega=\Omega(\Omega V F) \Omega$ we see that it is not in $\Pi$, since otherwise $\Pi$ would contain $V=F$.

Lemma 30 The interval $\left[\Pi, \Pi^{\prime}\left[\right.\right.$ is omitted by all p.o $o_{\perp} \lambda$-models.

Proof. Any p.o. model $\mathcal{M}$ of $\Pi$ satisfies $\Omega=\Omega \perp \perp \leq \Omega V F$, hence it also satisfies $\Omega=\Omega \Omega \Omega \leq \Omega(\Omega V F) \Omega \leq \Omega(\Omega V F)(\Omega V F)=\Omega$, hence $\Omega=\Omega(\Omega V F) \Omega$. Thus $T h(\mathcal{M}) \supseteq \Pi$ implies $T h(\mathcal{M}) \supseteq \Pi^{\prime}$.

Corollary 31 All the known classes $\mathcal{C}$ of $\lambda$-models are $2^{\omega}$-incomplete.

Note that, given a specific class $\mathcal{C}$, it is in general possible to find more natural intervals (ex: $\left[\lambda_{\beta \eta}, T o p\right]$ for $\mathcal{G}$, since no graph model is extensional). We will also see below that for each usual class $\mathcal{C}, \lambda \mathcal{T}-\lambda \mathcal{C}$ is $2^{\omega}$-broad. While Theorem 28 and its proof produce $2^{\omega}$-high intervals, which are furthermore independent of $\mathcal{C}$, and hence brings uniformity.

\section{Graph models.}

\subsection{Definition.}

For brevity we will confuse graph models and their webs, hence we simply define:

Definition 32 A graph model is a pair $(D, i)$, where $D$ is a non empty set and $i: D^{*} \times D \rightarrow D$ is a total injective function. 
Such a pair will also be called a total pair. It follows from the definition that $D$ is infinite. A total pair $(D, i)$ generates a reflexive cpo $\left(\mathcal{P}(D), \lambda_{i}, A_{i}\right)$, where $\lambda_{i}$ and $A_{i}$ are defined as follows:

$$
\lambda_{i}(g)=\{i(a, \alpha): \alpha \in g(a)\}
$$

The left inverse $A_{i} \in[\mathcal{P}(D) \rightarrow[\mathcal{P}(D) \rightarrow \mathcal{P}(D)]]$ of $\lambda_{i}$ (which allows to interpret application in the model) is defined by:

$$
A_{i}(d)\left(d^{\prime}\right)=\left\{\alpha \in D:\left(\exists a \subseteq d^{\prime}\right) i(a, \alpha) \in d\right\} .
$$

where $d, d^{\prime}$ are arbitrary subsets of $D$. When no ambiguity occurs we write $d d^{\prime}$ instead of $A_{i}(d)\left(d^{\prime}\right)$. The interpretation $|t|^{i}: \operatorname{Env}_{\mathcal{P}(D)} \rightarrow \mathcal{P}(D)$ of a $\lambda$-term $t$ with respect to $(D, i)$ is hence defined by induction by:

- $|x|_{\rho}^{i}=\rho(x)$

- $|t u|_{\rho}^{i}=\left\{\alpha:\left(\exists a \subseteq|u|_{\rho}^{i}\right) \quad i(a, \alpha) \in|t|_{\rho}^{i}\right\}$

- $|\lambda x . t|_{\rho}^{i}=\left\{i(a, \alpha): \alpha \in|t|_{\rho[x: a]}^{i}\right\}$

Since $|t|_{\rho}^{i}$ only depends on the value of $\rho$ on the free variables of $t$, we just write $|t|^{i}$ if $t$ is closed, and $|t|$ if furthermore $i$ is clear from the context.

\section{Example 33}

$$
\begin{aligned}
& |I|^{i}=|\lambda x . x|^{i}=\{i(a, \alpha) / \alpha \in a\} ;|K|^{i}=\{i(a, i(b, \alpha)) / \alpha \in a\} \\
& |\varepsilon|^{i} \equiv|\lambda x \lambda y . x y|^{i}=\left\{i(a, i(b, \alpha)) / \exists b^{\prime} \subseteq b\left(b^{\prime}, \alpha\right) \in a\right\} \\
& |\delta|^{i} \equiv|\lambda x . x x|^{i}=\{i(a, \alpha) / \alpha \in a a\} .
\end{aligned}
$$

Remark 34 It is easy to check that for all $t \in \Lambda^{0}$ and all $(D, i)$ we have:

$|t|^{i} \cap$ range $(i) \subseteq|\varepsilon t|^{i} \subseteq$ range $(i)$.

Recall that $\mathcal{G}$ (resp. $\mathcal{G}^{\text {den }}$ ) denotes the class of graph models (resp. whose web is countable); thus, all the models in $\mathcal{G}^{\text {den }}$ have cardinality $2^{\omega}$. The elements of $\lambda \mathcal{G}$ will be called graph theories for short.

\subsection{First equational and inequational properties.}

Lemma 35 No graph model is extensional.

Proof. In all graph models $(D, i)$ we have $i(\{i(\emptyset, \alpha)\}, i(\{\alpha\}, \alpha)) \in|\varepsilon|-|I|$.

This first and old observation happens to be the simplest instance of a general, and much less trivial, recent result, that we will state in Section 6.1.

Lemma 36 In all $(D, i)$ and for all $t \in \Lambda^{0}$ we have:

(i) $|I| \subset|\varepsilon|$ if and only if $i$ is onto.

(ii) $|t| \subseteq|\varepsilon t|$ if and only if $|t| \subseteq$ range $(i)$. 
Proof. (i) is easy to check, and left as an exercise, and (ii) follows easily from Remark 34.

Lemma 37 (Kerth 1995) In all graph models $(D, i)$ we have:

(i) $\left|\delta_{3}\right| \neq D$ and,

(ii) if $\alpha \in\left|\Omega_{3}\right|$, then $\alpha=i(\bar{a}, \alpha)$ for some $n>0$ and $a_{1}, \ldots, a_{n} \subseteq\left|\delta_{3}\right|$.

Proof. (i) It is easy to see that $\left|\delta_{3}\right|$ contains no element of the form $i(\emptyset, \gamma)$. The proof of (ii), which can be found in [33, Example 5.3.7], is a non straightforward, but not difficult, exercise.

Corollary 38 (Kerth 1995) No graph model satisfies $\Omega_{3} u=I\left(\right.$ for any $\left.u \in \Lambda^{0}\right)$.

Proof. Let $(D, i)$ be a graph model, and let $\gamma \in D-\left|\delta_{3}\right|$. We have $i(\{\gamma\}, \gamma) \in$ $|I|$. If $i(\{\gamma\}, \gamma) \in\left|\Omega_{3} u\right|$ then there is $a \subseteq|u|$ such that $\gamma^{\prime}=i(a, i(\{\gamma\}, \gamma)) \in\left|\Omega_{3}\right|$. By (ii) of the above lemma and using the injectivity of $i$ we get $\gamma \in\left|\delta_{3}\right|$, which is a contradiction.

This corollary, which remains true for $\mathcal{K}$ (and beyond) shows a concrete limitation of $\mathcal{G}$ and $\mathcal{K}$. Indeed $\Omega_{3} I$ is an easy term; this was proved syntactically by Jacopini and Zilli in 1985 [28], but was only given recently a semantic proof, by Honsell \& al. [1], who built, for each closed $t$, a filter model of $\Omega_{3} I=t$. Now, the above corollary shows that there can exist no semantic proof via graph models or models of $\mathcal{K}$, in contrast to the case $\Omega$, since $\Omega_{3} I=I$ is satisfied in no such model. This essentially answers Question 5 of [9, Section 6.3, p.152].

From Lemma 37 Bucciarelli and Salibra also derived the following interesting consequences; the first one is immediate, also using Lemma 36.

Corollary $39\left|\Omega_{3}\right| \subseteq\left|\varepsilon \Omega_{3}\right|$ is true in all graph models.

Combining this property with Theorem 26 we get:

Corollary 40 (Bucciarelli and Salibra 2004) [15] $\mathcal{G}$ omits $\lambda_{\beta}$.

Proposition 41 (Bucciarelli and Salibra 2004) [15] Each sensible graph model interprets all the closed unsolvable terms by the empty set.

Proof. It is enough to show that if a graph model $(D, i)$ satisfies $v=\lambda x . v$ and $v \subseteq \Omega_{3}$ for some closed term $v$, then $(D, i)$ interprets $v$ by $\emptyset$. Suppose $\alpha \in|v|$, then also $i(a, \alpha) \in|v| \subseteq\left|\Omega_{3}\right|$, for all $a \in D^{*}$. By Lemma 37, $i(a, \alpha)=i(b, \beta)$ for some $b \subseteq\left|\delta_{3}\right|$ and some $\beta$; hence $a=b \subseteq\left|\delta_{3}\right|$; since $a$ is arbitrary we would have $D \subseteq\left|\bar{\delta}_{3}\right|$, a contradiction.

The class $\mathcal{G}_{\text {lazy }}$ of lazy graph models differs from $\mathcal{G}$ by a small variation in the definition of $\lambda_{i}$, which works only if $i$ is non surjective: fix $\gamma \in D-\operatorname{range}(i)$, then define $\lambda_{i}^{\prime}(f) \equiv \lambda_{i}(f) \cup\{\gamma\}$. It is easy to check that $\left(\mathcal{P}(D), \lambda_{i}^{\prime}, A_{i}\right)$ is still a reflexive cpo. The first and simplest example of a lazy graph model is the lazy variation $\mathcal{E}_{\text {lazy }}$ of $\mathcal{E}$ introduced by Longo in [40]; as already noticed $T h\left(\mathcal{E}_{\text {lazy }}\right)=L T$, hence $L T$ is representable in $\mathcal{G}_{\text {lazy }}$. 


\subsection{Building graph models.}

There are only two methods for building graph models, and both consist in completing a partial pair into a total one, either freely or by forcing. When applied to total pairs both methods leave them unchanged. There is a also a product construction, called $\mathcal{G}$-product below, which is a particular case of free completion.

All these methods can be extended to the other classes of webbed models, with more or less ease (see Section 7 ). The systematic extension of the free completion method to $\mathcal{K}$ was written down in [9], but some particular cases had been previously worked out by several authors, to begin with [38] (cf. [9]).

\subsubsection{Partial pairs.}

Definition $42 A$ partial pair is a pair $(P, j)$ where $P$ is a non empty set and $j$ is a partial (possibly total) injection from $P^{*} \times P$ to $P$, written $j: P^{*} \times P \rightarrow P$.

The simplest example of a partial pair is $(P, \emptyset)$, and, of course, any total pair is a partial pair. In the examples $j$ will be described by its graph (in the usual sense). A crucial dichotomy occurs between the pairs which we call positive, and the other ones. A definition of positive pair, in full generality, was proposed in $[9$, p.125], and it was left to the reader to write down alternative formalizations. Such a (more intuitive, but possibly less general) formalization is the following.

Definition $43(P, j)$ is positive if there exists a function $v: P \rightarrow\{+,-\}$ such that $((a, \alpha), \beta) \in \operatorname{graph}(j)$ implies $v(\beta)=v(\alpha)$ and implies $v(\beta)=-v(\gamma)$ for all $\gamma \in a$.

\subsubsection{The free completion method.}

This method, just called "completion" in [9], and "canonical completion" elsewhere, generalizes the construction of $\mathcal{E}$. It was introduced by Longo in [40], who also proved that the graph model $P_{\omega}$ is isomorphic to the free completion of the pair $(\{0\},\{(\emptyset, 0), 0\})$, in a sense we will not make precise here. It was then used on a larger scale by Kerth (see Sections 6.2 and 6.3), who also transferred the method to other semantics [33, 35, 37], and it was also used recently by Bucciarelli-Salibra in $[14,15]$. Free refers here to the fact that the graph model $(D, i)$ is built in an inductive and canonical way from the partial pair $(P, j)$ we start with, as freely as possible.

Definition 44 The free completion of the partial pair $(P, j)$ is the total pair $(D, i)$ where $D$ is the smallest set such that

$$
D=P \cup\left(\left(D^{*} \times D\right)-\operatorname{dom}(j)\right) .
$$

and $i$ is defined by

$$
\begin{aligned}
& i(a, \alpha)=j(a, \alpha) \text { if }(a, \alpha) \in \operatorname{dom}(j) \\
& i(a, \alpha)=(a, \alpha) \text { otherwise }
\end{aligned}
$$


Hence $D$ is countable and could be defined as the union of an increasing sequence of sets $D_{n}$, while $i$ is (globally) defined at the end. We now recall the key examples (others can be found in [9]).

Example 45 (Engeler's model) $\mathcal{E}_{P}$ is the free completion of the (positive) pair $(P, \emptyset)$; in this case $i$ is just inclusion. Note that each element of $\mathcal{E}_{P}$ can uniquely be written as $(\bar{a}, \alpha)$ for some $\alpha \in P$ and $\bar{a} \in D^{*<\omega}$.

Example 46 The model $\mathcal{P}_{P}$ is the free completion of the (positive) partial pair $\left(P, j_{P}\right)$ where $\operatorname{dom}\left(j_{P}\right)=\{\emptyset\} \times P$ and $j_{P}(\emptyset, p)=p$ for all $p \in P$.

Example 47 The model $\mathcal{P}_{P}^{\prime}$ is defined as above except that $\operatorname{dom}\left(j_{P}\right)=\{(\{p\}, p)$ : $p \in P\}$ and $j_{P}(\{p\}, p)=p$ for all $p \in P$.

Definition 48 Let us call positive graph theories the theories of graph models which can be obtained by freely completing a positive pair.

Formally, the definition of $\mathcal{P}_{P}$ and $\mathcal{P}_{P}^{\prime}$ are similar, and the web of $\mathcal{P}_{P}^{\prime}$ is as simple as for $\mathcal{P}_{P}$. However the two families behave quite differently, as we will see soon; in particular all the models $\mathcal{P}_{P}$ are sensible, while in $\mathcal{P}_{P}^{\prime}$ we have: $|\Omega|=P$ and $|\Omega t|_{\rho}=P \cap|t|_{\rho}[26]$. The key structural difference between the two families is that $\mathcal{P}_{P}$ is generated by a positive pair, which is not the case for $\mathcal{P}_{P}^{\prime}$.

Indeed, if a model happens to be the free completion of a positive pair, then we have a (uniform) control on its theory. First it can be proved that positive graph theories are sensible (cf. [9, p.125]); the more direct way to prove it is to use a reducibility method (in Tait's spirit) directly in the model (as it is done there). Second, if furthermore one can apply the strong approximation theorem ${ }^{1}$ in the spirit of Hyland and Wadsworth [27][56], which is the case for the $\mathcal{E}_{P}{ }^{\prime} s$ and the $\mathcal{P}_{P}{ }^{\prime} s$ (in particular for $P_{\omega}$ ) then $T h(D, i)$ is completely known, and equal to $B T$. An open problem, which we raised in [9], and for which we have only partial positive answers yet, is whether this is always true for models generated by positive pairs. We will return to this point later on.

Remark 49 The theory of $\mathcal{E}_{P}$ (resp. $\mathcal{P}_{P}$ and $\mathcal{P}_{P}^{\prime}$ ) is independent of $P$.

Remark 50 Viewing $P_{\omega}$ as $\mathcal{P}_{\{0\}}$ makes it easier to study $T h\left(P_{\omega}\right)$.

The reason why $P_{\omega}$ and $\mathcal{P}_{\{0\}}$ can be proved to have the same theory is that we have a good notion of isomorphism between webs. The definition is as follows: let us say that $\theta: D \rightarrow D^{\prime}$ is a morphism between $(D, i)$ and $\left(D^{\prime}, i^{\prime}\right)$ if for all $a, \alpha$ we have that $\theta(i(a, \alpha))=i^{\prime}\left(\theta^{+}(a), \theta(\alpha)\right)$; by definition $\theta$ is an isomorphism if it is furthermore a bijection (then its inverse is also an isomorphism). This notion of isomorphism is good in the sense that isomorphic webs generate models with the same theory; but the mere notion of morphism happens to be disappointing (one would of course like to induce an inclusion of theories).

\footnotetext{
${ }^{1}$ The more general but weaker Approximation Theorem that can be found in [26], although helpful, is not enough.
} 


\subsubsection{The graph-product construction.}

Definition 51 [14] The $\mathcal{G}$-product of the family $\left(D_{e}, i_{e}\right)_{e \in E}$ of graph models, where the $D_{e}$ are supposed to be disjoint, is the free completion $\left(D_{E}, i_{E}\right)$ of $\left(\cup D_{e}, \cup i_{e}\right)$, and hence a graph model.

Note that $\mathcal{G}^{\text {den }}$ is only closed under countable products (i.e. $E$ should be countable). In the sequel it will be clear that the families we chose can be supposed to consist of disjoint sets without loss of generality. Finally, in the rest of this subsection we use freely the notations of the above definition.

In order to make clearer the presentation of the two following propositions, which express the key technical properties of the $\mathcal{G}$-product, and that of their consequences, we will state them separately. However they are not independent, in the sense that their only known proof is global and by mutual induction on $t$, once the family $\left(f_{e}\right)_{e \in E}$ has been exhibited [14].

Proposition 52 (Bucciarelli-Salibra 2003) For all $t \in \Lambda^{\circ}$ we have:

$$
|t|^{i_{e}}=|t|^{i_{E}} \cap D_{e}
$$

Corollary $53 T h\left(D_{E}, i_{E}\right) \subseteq \underset{e \in E}{\cap} T h\left(D_{e}, i_{e}\right)$.

Corollary 54 Any $\mathcal{G}$-product of semi-sensible graph models is semi-sensible.

Proposition 55 (Bucciarelli-Salibra 2003) There is a family $\left(f_{e}\right)_{e \in E}$ of functions $f_{e}: D_{E} \rightarrow D_{E}$ such that:

(i) $D_{E}$ is the (disjoint) union of the $f_{e}^{-1}\left(D_{e}\right), e \in E$;

(ii) $f_{e}\left(|t|^{i_{E}}\right) \subseteq|t|^{i_{E}}$.

Corollary 56 [15] Any $\mathcal{G}$-product of sensible graph models is sensible.

Proof. By Proposition 41 we have to prove that all unsolvables $v$ are interpreted by $\emptyset$ in the product. Suppose $\alpha \in|v|^{i_{E}}$; by (i) of Proposition 55, there is an $e \in E$ such that $f_{e}(\alpha) \in D_{e}$, and by (ii) of the same proposition $f_{e}(\alpha) \in|v|^{i_{E}}$. Thus $f_{e}(\alpha) \in|v|^{i_{e}}$ by Proposition 52, which contradicts the sensibility of $\left(D_{e}, i_{e}\right)$, using Proposition 41 once more.

\subsubsection{The forcing completion method.}

This method originates in Baeten-Boerboom [3], where it is used for proving the "easiness" of $\Omega$. In the simpler presentation proposed by Zylberajch [57] (see e.g. [10] for a published proof), one starts from a partial pair $(D, \emptyset)$, where $D$ is an infinite countable set, and builds by induction a total $i: D^{*} \times D \rightarrow D$, hence a graph model $(D, i)$. Thus, here, $D$ is fixed during the construction. Another difference is that the inductive construction itself depends on the consistency problem we are interested in, and moreover it exploits heavily the fact that the interpretation of $\Omega$ can be quite freely constrained. The method was generalized to families of terms having a similar behavior as $\Omega$ by Zylberajch [57], and then transferred by other authors to other classes of models and other semantics (cf. Section 7.2). One can also have to start from pairs $\left(D, p_{0}\right)$, with small constraints on $p_{0}$. 


\subsubsection{Comparing free and forcing completions.}

Besides the differences mentioned above it is worth noting the following points where the two methods behave differently.

Control or non control over $T h(\mathcal{M})$ ? Forcing never gives us control on the whole of $T h(\mathcal{M})$, even if we start from a positive partial web. A first consequence is that, even though $(D, \emptyset)$ is a positive web, it is likely that no model built by completing $(D, \emptyset)$ by forcing will be sensible, and most of them are furtherclearerly non semi-sensible; thus forcing can't be used to produce sensible theories and to study $\lambda \mathcal{G}^{s}$ and $\lambda \mathcal{T}^{s}$. A second consequence is that forcing can't be used to study the height of $\lambda \mathcal{G}$ since, given two models $\mathcal{M}, \mathcal{M}^{\prime}$ built by forcing, we will never be able to prove that $T h(\mathcal{M}) \subseteq T h\left(\mathcal{M}^{\prime}\right)$.

Preserving recursivity or not. From a recursive partial web, free completion builds a recursive total web (hence a graph model that could be viewed as a reasonable intersection type system (cf.[9])), while non trivial forcing always create a non recursive web.

Possible cardinalities of the webs. Forcing produces models with countable webs, while free completion can be used for building webs of any infinite cardinality.

Mass production of models. Free completion allows for mass production of non isomorphic graph models (and might probably allow for $2^{\omega}$ non isomorphic sensible graph models), usual forcing does not. However, and as we will see in the next subsection, it is possible to extend the method so that it becomes very easy to create $2^{\omega}$ graph models with pairwise inconsistent theories. Such theories are neither semi-sensible nor recursively enumerable. As already mentioned, this generalization has also other interesting applications to $\lambda \mathcal{T}$.

\subsubsection{Generalized forcing.}

Berline and Salibra [10] generalized recently the forcing method in three directions. First they noticed that the method works with other "operators" than forcing (but this is inessential for our concern here), second that it can be applied to terms with parameters in $\cup\left[P(D)^{n} \rightarrow P(D)\right]$, where the union is taken over $n \geq 0$, which opens the way to a lot of potential applications and, third, that it allows us to treat (finite and) infinite sequences of closed terms instead of a single term $t$ as above. Given a set $D$ we define the set $\Lambda_{D}$ of $D$-generalized $\lambda$-terms with the same inductive definition as for $\Lambda$, except that $\Lambda_{D}$ is furthermore closed under all continuous functions on $P(D)$ of arbitrary arity $n \geq 0$. In other words we add the clauses: $P(D) \subseteq \Lambda_{D}$ and, if $f \in\left[P(D)^{n} \rightarrow P(D)\right]$, $n \geq 1$, and if $t_{1}, \ldots, t_{n} \in \Lambda_{D}$ then $f\left(t_{1}, \ldots, t_{n}\right) \in \Lambda_{D}$.

As a corollary of the main results proved in [10] we get: 
Theorem 57 (Berline-Salibra 2004) There is a sequence of unsolvable terms $\left(v_{k}\right)_{k<\omega} \in \Lambda^{0}$ such that, for all countable sets $D$ and all sequences $\left(r_{k}\right)_{k \in \omega} \in \Lambda_{D}{ }^{\circ}$ there is $i: D^{*} \times D \rightarrow D$ such that the graph model $(D, i)$ satisfies $v_{k}=r_{k}$ for all $k$.

The simplest infinite such sequence is $v_{k}=\Omega \pi_{k, k}$, where $\pi_{k, k} \equiv \lambda x_{1} \ldots \lambda x_{k} . x_{k}$; the result is however far from straightforward. Note that the $v_{k}$ 's are, anyway, necessarily easy terms.

\subsection{Applications to $\lambda \mathcal{T}$.}

We now present two direct applications of generalized forcing to $\lambda \mathcal{T}$, which furthermore give evidence that the method is very promising, and should produce many other interesting consequences in the future. First we prove, semantically, that $\lambda \mathcal{T}$ is $2^{\omega}$-broad.

Proposition 58 (Berline-Salibra 2004) $\lambda \mathcal{G}^{d e n}$ is $2^{\omega}$-broad, hence $\lambda \mathcal{T}$ also.

Proof. Let $s=\left(t_{k}\right)_{k \in \omega}$ be an infinite sequence of closed normal terms such that $t_{n}=t_{m}$ is consistent only if $m=n$, for example $s$ is the sequence of Church integers. Thanks to Theorem 57 for each permutation $\sigma$ of $\omega$ there is an $i_{\sigma}$ such that the graph model $G_{\sigma} \equiv\left(D, i_{\sigma}\right)$ satisfies $v_{k}=t_{\sigma(k)}$ for all $k$. Now, by Böhm's theorem, $t_{m}=t_{n}$ is inconsistent if $m \neq n$, thus $T h\left(G_{\sigma}\right)$ and $T h\left(G_{\sigma^{\prime}}\right)$ are inconsistent if $\sigma \neq \sigma^{\prime}$.

Proposition 59 There is a finitely axiomatizable theory $T$ such that $[T, T o p]$ is a distributive lattice.

Proof. Given $D$, intersection $\cap$ and union $\cup$ are two binary continuous functions on $\mathcal{P}(D)$. Let $r_{1}, r_{2} \in \Lambda_{D}$ be defined by: $r_{1} \equiv \lambda x \lambda y .(x \cap y)$ and $r_{2} \equiv \lambda x \lambda y .(x \cup y)$. Let $G \equiv(D, i)$ be any graph model satisfying $v_{1}=r_{1}$ and $v_{2}=r_{2}$, where $v_{1}, v_{2}$ are as in Theorem 57. Since $\mathcal{P}(D)$ is a (distributive) lattice, $G$ also satisfies the equations between terms of $\Lambda$ which express that we have a (distributive) lattice when $v_{1}, v_{2}$ play the role of join and meet (for example distributivity itself is expressed by the equation $\left.v_{1} x\left(v_{2} y z\right)=v_{2}\left(v_{1} x y\right)\left(v_{1} x z\right)\right)$. Let $T$ be the $\lambda$-theory generated by these equations. We have proved that $T$ is consistent and that there are two terms which make its term model $\Lambda / T$ a (distributive) lattice. Thus, the lattice of congruences of $\Lambda / T$ is the lattice of congruences of a lattice, and hence a distributive lattice, by [42, Theorem 2.50 , and the remark following the proof of this theorem]. Since $[T, T o p]$ is isomorphic to this lattice, it is hence also a distributive lattice.

By slightly modifying the proof we have here for free that $[T, T o p]$ is $2^{\omega_{-}}$ broad: just work as in the proof of Proposition 58 , but with sequences $r_{1}, r_{2}, t_{1}, \ldots, t_{n}, \ldots$ where $r_{1}$ and $r_{2}$ are as above (and untouched by the permutations $\sigma$ ). This also proves point (i) of the following remark; for proving its point (ii) one just has to use sequences $r_{1}, r_{2}, \hat{n}$. 
Remark 60 (i) There exist $2^{\omega}$ pairwise inconsistent theories $T$ such that $[T, T o p]$ is a distributive lattice.

(ii) There exist $\omega$ pairwise inconsistent and finitely axiomatizable theories $T$ such that $[T, T o p]$ is a distributive lattice.

Of course, one may wonder now whether $\lambda \mathcal{T}$ is the union of such distributive lattices.

\subsection{Graph models and strong surjective pairing.}

A $\lambda$-model $\mathcal{M}$ models surjective pairing (SP) if there are $c, p_{1}, p_{2} \in \mathcal{M}$ such that the equations $p_{1}(c x y)=x, p_{2}(c x y)=y$ and $c\left(p_{1} z\right)\left(p_{2} z\right)=z$ are satisfied in the model. It is well-known that the term models of $\lambda_{\beta}$ and $\lambda_{\beta \eta}$ do not model surjective pairing (Klop 1980, cf.[4, Ex. 15.4.4]). On the contrary, it is easy to see that any proper model $\mathcal{M}=(\mathcal{D}, A, \lambda)$ living, say, in $\mathcal{C}_{\text {cont }}$, and such that $\mathcal{D} \simeq \mathcal{D} \times \mathcal{D}$ models surjective pairing, since then one can indeed take $c=\lambda(f)$, $p_{1}=\lambda\left(\pi_{1}\right)$ and $p_{2}=\lambda\left(\pi_{2}\right)$, where $f: \mathcal{D} \times \mathcal{D} \rightarrow \mathcal{D}$ and $\left(\pi_{1}, \pi_{2}\right): \mathcal{D} \rightarrow \mathcal{D} \times \mathcal{D}$ are any pair of inverse isomorphisms. Note that all graph models have this property, since for all infinite set $D$ we have $\mathcal{P}(D) \times \mathcal{P}(D) \simeq \mathcal{P}(D)$; to see this note that clearly $\mathcal{P}(D) \simeq \mathcal{P}\left(D_{1}\right) \times \mathcal{P}\left(D_{2}\right)$ for any good partition $D=D_{1} \cup D_{2}$ of $D$, where good means: into two subsets of the same cardinality as $D$.

$\mathcal{M}$ models strong surjective pairing (Strong SP) if $\mathcal{M}$ models SP in such a way that it furthermore satisfies: $p_{1} x y=p_{1}(x y)$ and $p_{2} x y=p_{2}(x y)$. It was proved in [38] (and rediscovered independently in [19]) that $\mathcal{D}_{\infty}$ models Strong SP. Then Jiang proved in her thesis [29][30] that one could build, by forcing, extensional models of $\mathcal{K}$ which satisfied furthermore much stronger constraints.

We wish to point out here that (a lot of) graph models can also model Strong SP, and that the simplest model of Strong SP is Engeler's model $\mathcal{E}_{P}, P$ infinite (the simplest extensional models being $\mathcal{D}_{\infty}$ or $\mathcal{P}_{\infty}$, when presented as $\mathcal{K}$-models, and also relative to an infinite $P$ ). In fact the following proof is just the relevant simplification of Krivine's proof for $\mathcal{D}_{\infty}$. Jiang's variants of strong pairing could also have been modelled more simply within graph models, at the price of loosing extensionality.

Proposition 61 If $P$ is infinite then $\mathcal{E}_{P}$ models strong surjective pairing.

Proof. Let $P$ be an infinite set, let $(D, \subseteq)$ the web of $\mathcal{E}_{P}$, let $P=P_{1} \cup P_{2}$ be a good partition of $P$, and finally let $D_{i}=\left\{(\bar{a}, \alpha): \alpha \in P_{i}\right\}$ for $i=1,2$ (thus $D=D_{1} \cup D_{2}$ is also a good partition of $D$ ). Let now $\varphi_{i}: P \rightarrow P_{i}$ be two bijections, let $\bar{\varphi}_{i}: D \rightarrow D$ be defined by: $\bar{\varphi}_{i}(\bar{a}, \alpha)=\left(\bar{a}, \varphi_{i}(\alpha)\right)$ if $\alpha \in P$, let $\pi_{i}=\bar{\varphi}_{i}^{-}: \mathcal{P}(D) \rightarrow \mathcal{P}(D)$ and let $f: \mathcal{P}(D) \times \mathcal{P}(D) \rightarrow \mathcal{P}(D)$ be defined by $f\left(d_{1}, d_{2}\right)=\bar{\varphi}_{1}^{+}\left(d_{1}\right) \cup \bar{\varphi}_{2}^{+}\left(d_{2}\right)$. Then it is clear that $f$ and $\left(\pi_{1}, \pi_{2}\right)$ are inverse isomorphisms, and it is furthereasier to check that $p_{i}=\lambda\left(\pi_{i}\right)$ satisfies, for $i=1,2$, the further condition needed for Strong SP.. 


\section{The structure of $\lambda \mathcal{G}$.}

In order to have a clearer picture of $\lambda \mathcal{G}$ we continue to feel free of any respect to historical chronology. As announced, we will see that extending some of the results obtained for $\mathcal{G}$ to $\mathcal{G}^{s}$, and hence from $\lambda \mathcal{T}$ to $\lambda \mathcal{T}^{s}$ ranges, when possible, from straightforward to highly difficult.

The links between $\lambda \mathcal{G}$ and $\lambda \mathcal{G}^{\text {den }}$ are simpler, in a sense, since all the results proved in this paper for $\lambda \mathcal{G}$ also hold for $\lambda \mathcal{G}^{\text {den }}$, and with the same proofs, because one only uses models built by forcing or free completion of finite or countable partial webs, and hence in $\mathcal{G}^{\text {den }}$. However the following natural question is still open, as far as we know.

Problem 12 Does $\lambda \mathcal{G}=\lambda \mathcal{G}^{\text {den }}$ ?

\subsection{Least and largest elements of $\lambda \mathcal{G}$ and $\lambda \mathcal{G}^{s}$.}

The following lemma is a corollary of the proof of Proposition 58, which provides $2^{\omega}$ pairwise inconsistent graph theories, but it can also be given a direct and shorter proof, as below.

Lemma $62 \lambda \mathcal{G}$ and $\lambda \mathcal{G}^{\text {den }}$ have no greatest element.

Proof. Let $\mathcal{M} \in \mathcal{G}^{\text {den }}$ satisfying $\Omega=I$ (by forcing). Then $T h(\mathcal{E})$ and $T h(\mathcal{M})$ are incompatible, since $\Omega=\lambda x . \Omega$ is in $B T=T h(\mathcal{E})$, and the set $\{\Omega=I, \Omega=\lambda x \Omega\}$ is inconsistent.

The situation happens to be different for $\mathcal{G}^{s}$, as discovered by Bucciarelli and Salibra. The proof of the following theorem, which gives the key to a positive answer, and also generalizes the fact that no graph model is extensional, is quite technical and can be found in [15].

Theorem 63 (Bucciarelli and Salibra 2004)

All the equations of $H^{*}-B T$ are false in all graph models.

Corollary 64 (Bucciarelli and Salibra 2004 [15])

$\lambda \mathcal{G}^{s}$ and $\lambda \mathcal{G}^{s / 2}$ have a greatest element, which is BT in both cases.

Proof. Let $T \in \lambda \mathcal{G}^{s}$. Since $T$ is sensible we have $T \subseteq H^{*}$; then, since $T$ omits each of the equations of $H^{*}-B T$ we have $T \subseteq B T$.

Theorem 65 (Bucciarelli and Salibra 2003) [14] If $\mathcal{C} \subseteq \mathcal{G}$ is closed under countable $\mathcal{G}$-products, then $\lambda \mathcal{C}$ has a least element.

First proof. Let $E$ be the (countable) set consisting of the equations which fail to hold in some graph model. For each $e \in E$ let $\left(D_{e}, i_{e}\right)$ be a model where $e$ is false, let $T_{E}$ be the theory of the $\mathcal{G}$-product $\left(D_{E}, i_{E}\right)$ of this family, and finally let $T_{e}$ be the theory of $\left(D_{e}, i_{e}\right)$. By Theorem $53, T_{E} \subseteq \cap T_{e}$. Furthermore, if an equation doesn't belong to $T_{E}$, i.e. fails in $\left(D_{E}, i_{E}\right)$, which belongs to $\mathcal{C}$ 
by hypothesis, then it is one of the $e^{\prime} s$ and it fails in the corresponding $\left(D_{e}, i_{e}\right)$, hence it is not in $T_{e}$. Thus $T_{E}=\cap T_{e}$.

Second proof, simpler but assuming that $\mathcal{C}$ is closed under arbitrary products. For each $T \in \lambda \mathcal{C}$ take a model $\left(D_{T}, i_{T}\right)$ whose theory is $T$. The product $\left(D_{E}, i_{E}\right)$ has theory $T_{E} \subseteq \cap \lambda C$. Since $T_{E} \in \lambda \mathcal{C}$ we have $T_{E}=\cap \lambda C$.

Since $\mathcal{G}, \mathcal{G}^{s}$ and $\mathcal{G}^{s / 2}$ are closed under arbitrary products (by Corollary 54 and Proposition 56), and $\mathcal{G}^{\text {den }}$ under countable products, we get:

Corollary $66 \lambda \mathcal{G}, \lambda \mathcal{G}^{s}, \lambda \mathcal{G}^{s / 2}$ and $\lambda \mathcal{G}^{\text {den }}$ have least elements.

Let $T_{\min }$ and $T_{\min }^{s}$ be the least elements of $\lambda \mathcal{G}$ and $\lambda \mathcal{G}^{s}$. Since $T_{\min } \subseteq H$, $T_{\min }$ is semi-sensible and since $\lambda_{\beta} \notin \lambda \mathcal{G}$ (by Corollary 40) we have $\lambda_{\beta} \varsubsetneqq T$. Concerning $\lambda \mathcal{G}^{s}$, Bucciarelli and Salibra conjecture the following:

Conjecture 13 (Bucciarelli and Salibra 2004) [15] $T_{\min }^{s}=H$.

\subsection{Width and height of $\lambda \mathcal{G}$ and $\lambda \mathcal{G}^{\text {den }}$.}

We have already proved via forcing that $\lambda \mathcal{G}$ is $2^{\omega}$-broad. We now present some earlier results and proofs obtained via free completion, which are mainly due to Kerth (1994-1995). These proofs bring further information on $\lambda \mathcal{G}$ and $\lambda \mathcal{T}$, and also raise other kinds of questions. Everywhere $\mathcal{G}$ could be replaced by $\mathcal{G}^{\text {den }}$.

Theorem 67 (Kerth 1994). $\lambda \mathcal{G}$ is $2^{\omega}$-wide.

Sketch of proof. Kerth produced in [32] a family of non-positive pairs $\left(A_{W}, j_{W}\right)_{W \in \mathcal{P}(N)}$, and sets of equations $R_{W}=\left\{Y F_{n+1}=Y F_{2}: n \in W\right\}$, where $F_{n}=\lambda x . \lambda x_{1} \ldots \lambda x_{n} . x$, such that $\left(A_{W}, j_{W}\right)$ satisfies all the equations of $R_{W}$ and no equation of $R_{N}-R_{W}$. Since $R_{W} \subseteq R_{W^{\prime}}$ if and only if $W \subseteq W^{\prime}$ and since $(\mathcal{P}(N), \subseteq)$ contains $2^{\omega}$ pairwise incomparable sets (see the proof of Lemma 7$)$, we deduce immediately that there are $2^{\omega}$ pairwise incomparable graph theories.

These models are nonsensible, since all the $Y F_{n}$ are clearly unsolvable. Later on, Kerth produced in [33], on the same principles, another family of theories, for which he had the hope (finally fulfilled) that they could be proved to be sensible. In both cases, producing the pairs, the equations $R_{W}$, and checking that the models had distinct theories, even if not difficult, required non trivial observations and some computations. Let us observe that this is not anymore the case for our proof of Proposition 58.

Problem 14 Can we say something about the height of $\lambda \mathcal{G}$ ? 


\subsection{Width of $\lambda \mathcal{G}^{s}$.}

\subsubsection{Producing 2 sensible graph theories is difficult.}

From the remarks in Section 5.3.5 it follows that the only method available for producing sensible graph models is the free completion method. Completing freely positive pairs brings sensibility for sure; but how many sensible theories can we obtain that way? In [9, Question 1 of p. 153] we asked whether webbed models generated by positive partial webs necessarily satisfy the (strong) Approximation Theorem of Hyland and Wadsworth. If this is true then all positive graph theories contain $B T$. Positive partial results which we obtained with Salibra (article in preparation) argue for a positive answer, but we met hard resistance for going further. Anyway, in view of Theorem 63 and of its corollary, we can now rephrase our question as follows (in the case of graph models).

Conjecture $15 B T$ is the only positive graph theory.

If this can be proved, then the same arguments should work for proving that $L T$ is the only positive lazy graph theory, and it will also have analogues in other classes of webbed models.

The state of the art. At the moment, Salibra and the author have proved the following partial (unpublished) results which rather argue in favour of the conjecture. First, all positive graph models give the same interpretation to terms $t, t^{\prime}$ as soon as they have the same Böhm tree if this tree is almost hereditarily head closed (ahhc), which means that all but a finite number of its nodes are equal to $\perp$ or have the form $\lambda \bar{x}$. $y$ with $y \in \bar{x}$. Second, all positive graph models $(D, i)$ interpret the Curry and Turing fixed point combinators $Y$ and $\Theta$ by the least fixed point operator $\mathcal{Y}$ of the complete lattice $\mathcal{P}(D)$, and hence satisfies $Y=\Theta$. Note that all fixed point combinators have the same Böhm tree, which consists in one infinite branch where all nodes, except the root, are labelled by the same variable $y$, the root itself being labelled by $\lambda y . y$. This tree is hence (nearly) the simplest example of a non-ahhc tree, nevertheless we are yet unable to prove that all positive graph models interpret all the fixed point combinators by $\mathcal{Y}$, even if we can of course treat other fixed point combinators than $Y$ and $\Theta$; moreover our proofs are different in case of $Y$-like and $\Theta$-like trees..

Thus, for producing sensible graph theories different from $B T$ we are left with the free completion of non positive pairs, which explains retroactively why Kerth's task of producing $2^{\omega}$-sensible graph theories was so complex. Indeed, proving sensibility of models generated by non positive webs happens to be very difficult.

More generally, producing a non extensional sensible proper model whose theory is different from $B T$ is difficult. This problem concerns all the classes of $\lambda$-models, since forcing completion and free completion are the only systematic ways we know for building models concretely. Even the inverse limit construction can, in practice, always be presented as a free completion.

The conceptual interest of $\mathcal{G}$ here is that, because its exploration is easier, the problem could be met, isolated, stated, and hence understood, more easily. 


\subsubsection{But producing $2^{\omega}$ sensible graph theories is possible.}

Theorem 68 (Kerth 1995 plus David 1999) $\lambda \mathcal{G}^{s}$ is $2^{\omega}$-wide.

Sketch of proof. Two very difficult steps. First Kerth built $2^{\omega}$ graph models $G_{W}$ as the free completions of adequate non positive pairs $\left(A_{W}, i_{W}\right), W \in \mathcal{P}(N)$ [33][35] Then he reduced the proof that the $G_{W}$ were sensible (although the pair was not positive) to a syntactic conjecture on the head-normalization of nonsolvable terms, for which he gave much evidence. The second step was the proof of this conjecture, four years later, by R. David [17][18].

We can be more precise: Kerth's created pairs $\left(A_{W}, i_{W}\right)$ such that $G_{W}$ satisfies $Y F_{n}=\Theta F_{n}$ if and only if $n \in W$, where $Y$ and $\Theta$ are the Türing and Curry fixed point operators, and here $F_{n} \equiv \lambda x \cdot \lambda y_{1} \ldots \lambda y_{n} . \lambda z \cdot(z) x$. In particular $G_{W} \vDash Y \neq \Theta$ for all $W \neq \emptyset$.

Corollary $69 \lambda \mathcal{G} \cap[H, B T]$ is $2^{\omega}$-wide.

Proof. By Theorem 68 and Corollary 64.

Corollary 70 There are $2^{\omega}$ theories of proper models included in $[H, B T]$.

This corollary largely answers positively Question 2 in [9, p. 151].

However the following questions remain open, since forcing essentially builds non sensible models, and they should be difficult.

Problem 16 Is $\lambda \mathcal{G}^{s} 2^{\omega}$-broad?

Problem 17 Is there a (large) distributive lattice of sensible theories?

Problem 18 Can we get information about the height of $\lambda \mathcal{G}^{s}$ ?

\section{The other $\lambda \mathcal{C}^{\prime} s$.}

\subsection{The other key classes of models.}

All the classes of models presented below live within one of the three main semantics (cf. Section 7), and are classes of webbed models (see [9] for a more detailed presentation of each of them). The methods used for building graph models or for proving positive results about $\mathcal{G}$ can be adapted to these other classes $\mathcal{C}$, at a cost which depends on $\mathcal{C}$.

- The simplest subclass of $C_{\text {cont }}$, strictly including $\mathcal{G}$, is the class $\mathcal{K}$ of $K$ models, which was isolated in [38]. The interest of $\mathcal{K}$ over $\mathcal{G}$ is that it contains (plenty of) extensional models. In particular, Scott's $\mathcal{D}_{\infty}$ and Park's $\mathcal{P}_{\infty}$ live in $\mathcal{K}$, and their description as K-models is much simpler than their prior descriptions, e.g. as inverse limits, and considerably simplifies their study. Finally, it is worth noting that $\mathcal{D}_{\infty}$ and $\mathcal{P}_{\infty}$ are respectively the extensional completion of the graph models $\mathcal{P}_{P}$ and $\mathcal{P}_{P}^{\prime} \in \mathcal{G}$, a process 
which is presented in $\left[9\right.$, p.130]. To be accurate, $\mathcal{D}_{\infty}$ and $\mathcal{P}_{\infty}$ are also built starting from a set $P$, and hence should also be indexed by $P$. This notation is somewhat misleading but respects the traditional notation for Scott's and Park's models.

More general, and hence more complex, classes of webbed models living in $\mathcal{C}_{\text {cont }}$ were introduced and used in the literature (ex: filter-models, information systems, and pcs-models), which are also surveyed and compared in [9]. As already noticed, even though $\lambda \mathcal{C}_{\text {cont }} \supsetneq \lambda \mathcal{K}$, it is exceptional that we have to go beyond $\mathcal{K}$ for proving things about $\lambda \mathcal{C}^{\text {cont }}$.

- The key subclass of $\mathcal{C}_{s t}$ is the class $\mathcal{G}_{\text {coh }}$ of reflexive coherent spaces, or $G$-models ("G" for "Girard").

- The key subclass of $\mathcal{C}_{\text {sts }}$ is the class $\mathcal{G}_{\text {hycoh }}$ of Ehrard's reflexive hypercoherences, or $H$-models ( $H$ " for "hypercoherence").

\subsubsection{Comparison with $\mathcal{G}$ and $\mathcal{K}$.}

The classes $\mathcal{G}_{\text {coh }}$ and $\mathcal{G}_{\text {hycoh }}$ are the analogues of $\mathcal{G}$ in their respective semantics, in the following sense. First $\mathcal{G}, \mathcal{G}_{c o h}$ and $\mathcal{G}_{\text {hycoh }}$ are the simplest classes of webbed models one can respectively find in $\mathcal{C}_{\text {cont }}, \mathcal{C}_{\text {st }}$ and $\mathcal{C}_{\text {sts }}$. Second, the definitions of $\mathcal{G}_{c o h}$ and $\mathcal{G}_{\text {hycoh }}$ can be seen as the variants of the definition of $\mathcal{G}$ adapted to $\mathcal{C}_{s t}$ and $\mathcal{C}_{s t s}$. On the other hand, $\mathcal{G}_{\text {coh }}$ and $\mathcal{G}_{\text {hycoh }}$ can also be viewed as the analogues of $\mathcal{K}$, since they are rich in extensional models, while $\mathcal{G}$ contains none. A last remark is that the definition and study of $\mathcal{G}_{\text {hycoh }}$ is significantly more complicate than the other two ones.

\subsubsection{Analogues of the key models of $\mathcal{G}$ and $\mathcal{K}$ in $\mathcal{G}_{c o h}$ and $\mathcal{G}_{\text {hycoh }}$.}

The constructions of $\mathcal{E}, \mathcal{P}_{P}, \mathcal{P}_{P}^{\prime} \in \mathcal{G}$ and of $\mathcal{D}_{\infty}, \mathcal{P}_{\infty} \in \mathcal{K}$ can be mimicked in both $\mathcal{G}_{\text {coh }}$ and $\mathcal{G}_{\text {hycoh }}$.

Notation 71 When the construction of a model $\mathcal{M}$ of $\mathcal{G}$ or $\mathcal{K}$ can be mimicked in $\mathcal{G}_{\text {coh }}$ (resp. $\mathcal{G}_{\text {hycoh }}$ ) we denote its analogue by $\mathcal{M}^{\text {st }}$ (resp. $\mathcal{M}^{\text {sts }}$ ).

Note that $\mathcal{M}^{s t}$ and $\mathcal{M}^{\text {sts }}$ need not exist and, when they do, they need not have the same equational theory as $\mathcal{M}$, although this also can happen. Examples of both situations will be given below.

\subsection{Transferring the completion methods.}

Free and forcing completions can be extended to $\mathcal{K}, \mathcal{G}_{c o h}$, and $\mathcal{G}_{\text {hycoh }}$. This often allows one to extend the positive results which have been proved for $\lambda \mathcal{G}$ to the three other classes, and hence to get new information on $\lambda \mathcal{T}$. However, the notion of web and that of a partial web is more complex than for graph models, for which partial webs are just partial pairs, as defined earlier. For example, the web of a $K$-model has the shape $(D, \leq, i)$, where $\leq$ is a preorder on $D$, and 
$i$ and $\leq$ have to satisfy some compatibility condition; the notion of partial web is then defined accordingly (and the cpo is now the complete lattice $\mathcal{S}(D)$ of all downward closed subsets of $(D, \leq)$, still ordered by inclusion, instead of $\mathcal{P}(D)$ ).

The free completion method in $\mathcal{K}, \mathcal{G}_{c o h}$, and $\mathcal{G}_{\text {hycoh }}$. Scott's model $\mathcal{D}_{\infty}$ and Park's model $\mathcal{P}_{\infty}$ are the extensional completions of the graph models $\mathcal{P}$ and $\mathcal{P}^{\prime}$ In particular $\mathcal{D}_{\infty}$ is generated by a positive partial web, while $\mathcal{P}_{\infty}$ is not; hence we can expect different theories, and we will see below that the difference between the behaviour of $\mathcal{D}_{\infty}$ and $\mathcal{P}_{\infty}$ is still deeper. It was shown in [26] that $\mathcal{P}_{\infty}$ is non sensible, and this is also the case of its graph analogue $\mathcal{P}^{\prime}$, for similar reasons.

The free completion method was systematically adapted to $\mathcal{G}_{\text {coh }}$ by Kerth [33][37] and to $\mathcal{G}_{\text {hycoh }}$ by Bastonero [5].

The forcing method in $\mathcal{K}, \mathcal{G}_{\text {coh }}$, and $\mathcal{G}_{\text {hycoh }}$. Y. Jiang was the first to build extensional models (of $\mathcal{K}$ ) by forcing $[29,30]$ (the aim was to produce models enjoying a strong notion of surjective pairing, combined with other properties). Later on Bastonero used forcing to build an extensional model $\mathcal{M} \in \mathcal{C}_{\text {cont }}$, such that $T h(\mathcal{M}) \notin \mathcal{C}_{s t} \cup \mathcal{G}_{\text {hyco }}[5]$.

The forcing method was systematically adapted to $\mathcal{G}_{c o h}$ (i.e. to the stable semantics) by Kerth [33, 36], and to $G_{h y c o h}$ (i.e. to the strongly stable semantics) by Bastonero and Kerth (unpublished).

\subsection{Transferring results from $\mathcal{G}$ to the $\mathcal{C}^{\prime} s$.}

Of course, only the positive results are transferable, in the good cases.

The first result shows that $B T$ and $H^{*}$ belong to $\lambda \mathcal{C}_{\text {cont }} \cap \lambda \mathcal{C}_{s t} \cap \lambda \mathcal{C}_{\text {sts }}$, which shows in particular that the three main semantics are non exclusive. This result has to be contrasted with the situation for Plotkin's $P C F$ [44], which is one of the best known typed $\lambda$-calculi. Indeed, the three "standard models" of $P C F$ in the main semantics have different, and even incomparable, equational theories [31][?, Chapter 9].

Theorem 72 (Bastonero and Gouy 1995-7) [7][8]

(i) $B T \in \lambda \mathcal{G} \cap \lambda \mathcal{G}_{\text {coh }} \cap \lambda \mathcal{G}_{\text {hyco }}$ and

(ii) $H^{*} \in \lambda \mathcal{K} \cap \lambda \mathcal{G}_{\text {coh }} \cap \lambda \mathcal{G}_{\text {hycoh }}$

Sketch of proofs. (i) Not only can the construction $\mathcal{E}$ be mimicked in $\mathcal{G}_{\text {coh }} \subseteq$ $\mathcal{C}_{s t}$ and $\mathcal{G}_{\text {hycoh }} \subseteq \mathcal{C}_{\text {sts }}$, but also the proof that $T h(\mathcal{E})=B T$, hence $B T \in$ $\lambda \mathcal{G} \cap \lambda \mathcal{G}_{\text {coh }} \cap \lambda \mathcal{G}_{\text {hycoh }}$ (Bastonero, [5] for $\mathcal{C}_{s t}$ and unpublished for $\mathcal{C}_{\text {sts }}$ ). (ii) The same is true for $\mathcal{D}_{\infty}$ (Gouy for $\mathcal{C}_{s t}$ [23][24], and with Bastonero for $\mathcal{C}_{\text {sts }}$ [7][8]), hence $H^{*} \in \lambda \mathcal{K} \cap \lambda \mathcal{G}_{\text {coh }} \cap \lambda \mathcal{G}_{\text {hycoh }}$. Only the results on $\mathcal{D}_{\infty}$ were published, since at the beginning we were focusing on extensional theories; the fact that the same worked for $\mathcal{E}$, of course with simpler proofs, was only emphasized later on (see [5]). 
Digression. What about $L T$, which is the third theory represented in $\mathcal{C}_{\text {games }}$ ? We did not check details but have a strong feeling that the construction of $\mathcal{E}_{\text {lazy }}$ in $\mathcal{G}_{\text {lazy }}$ can also be mimicked in (possibly slight variations of) $\mathcal{G}_{c o h}$ and $\mathcal{G}_{\text {hycoh }}$, as well as the proof that $T h\left(\mathcal{E}_{\text {lazy }}\right)=L T$, and hence that also $L T \in \lambda \mathcal{C}_{\text {cont }} \cap \lambda \mathcal{C}_{\text {st }} \cap \lambda \mathcal{C}_{\text {sts }}$.

The next theorem and remark show that $\lambda \mathcal{C}_{s t}$ and $\lambda \mathcal{C}_{s t s}$ are as "rich" as $\lambda \mathcal{C}_{\text {cont }}$. The proof uses forcing relative to $\mathcal{G}_{\text {coh }}$ (and $\mathcal{G}_{\text {hycoh }}$ ).

Theorem 73 (Kerth 1995) [33][37] $\mathcal{G}_{\text {coh }}$ is $2^{\omega}$-wide, and hence also $\lambda \mathcal{C}_{\text {st }}$.

Remark 74 It was later on noticed by Bastonero, that forcing, and hence the proof of this theorem could be adapted to $\mathcal{G}_{\text {hycoh }}$, but this remained unpublished. It is also likely that generalized forcing can be developed, at least for $\mathcal{G}_{\text {coh }}$, and hence that we can replace $2^{\omega}$-wide by $2^{\omega}$-broad.

\subsection{Relative positions of the $\lambda \mathcal{C}^{\prime} s$.}

We already know that $\lambda \mathcal{C}_{\text {cont }}, \lambda \mathcal{C}_{\text {st }}$ and $\lambda \mathcal{C}_{\text {sts }}$ are: $2^{\omega}$-wide, $2^{\omega}$-incomplete, and that their intersection contains $B T, H^{*}$ and probably $L T$; moreover we know that $\lambda \mathcal{T}-\lambda \mathcal{C}$ is $2^{\omega}$-high, for each of these $\mathcal{C}$. We show now that they are far from representing the same theories. First we state the problem we started from, when studying these questions.

Conjecture $19 \lambda \mathcal{C}_{\text {cont }}, \lambda \mathcal{C}_{\text {st }}$ and $\lambda \mathcal{C}_{\text {sts }}$ are pairwise incomparable (for inclusion).

Xavier Gouy and Olivier Bastonero got very close to proving this, since they succeeded to prove it for the triple $\mathcal{C}_{\text {cont }}, \mathcal{C}_{s t}$ and $\mathcal{G}_{\text {hycoh }}$. We will see below how, using generalized forcing one can improve this result.

Theorem 75 (Gouy and Bastonero 1996) [7][8]

(i) $\operatorname{Th}\left(\mathcal{P}_{\infty}^{s t}\right) \notin \lambda \mathcal{C}_{\text {cont }} \cup \lambda \mathcal{C}_{\text {sts }}$

(ii) $\operatorname{Th}\left(\mathcal{P}_{\infty}^{s t s}\right) \notin \lambda \mathcal{C}_{\text {cont }} \cup \lambda \mathcal{C}_{\text {st }}$.

Theorem 76 (Bastonero 95) [5, 6] One can build (by forcing) a $\lambda$-model $\mathcal{M} \in \mathcal{K}^{e}$ such that: $T h(\mathcal{M}) \notin \lambda \mathcal{C}_{s t} \cup \lambda \mathcal{G}_{\text {hycoh }}$.

Hint. Let $u=\Omega \lambda x . \Omega$ and $v=\Omega \lambda x \lambda y . \Omega$. There is a set $F$ consisting of seven equations and one inequation, relating $\Omega, u$ and $v$, and there is an equation: $t_{1}=t_{2}$, such that: no model of $\mathcal{C}_{s t} \cup \mathcal{G}_{\text {hycoh }}$ can satisfy $F^{\prime}=F \cup\left\{t_{1}=t_{2}\right\}$, but one can build, by forcing, a model $\mathcal{M} \in \mathcal{K}^{e}$ satisfying $F^{\prime}$. Since Bastonero was only concerned by extensionality, $F$ contains $I=\varepsilon$, and he had to use the extension of forcing which is adequate for $\mathcal{K}^{e}$. However the proof can easily be modified/simplified such that $\varepsilon=I$ is removed from $F$ and $\mathcal{M}$ is built by usual forcing in $\mathcal{G}$. We now give some information on the construction of $\mathcal{M}$ in this simpler setting. A triple $\left(p_{0}, h, D\right)$ is exhibited, in this order, such that: $p_{0}$ and $h$ are infinite, $D \supseteq C$, and $\left(D, p_{0}\right)$ is a partial pair satisfying the two easy 
constraints which make forcing possible from $\left(D, p_{0}\right)$; then a pair $(D, i), i \supseteq p_{0}$, is built by forcing such that $\mathcal{M}=(D, i)$ satisfies $\Omega=h$; but in fact $p_{0}$ was furthermore chosen in such a way that $\Omega=h$ implies $F^{\prime}$ in $\mathcal{M}$, which ends the (sketch of) the proof.

From the above theorem and from the observation that $\mathcal{M}$ could be built in $\mathcal{G}$, we derive immediately the following statements (note that none of them is a consequence of the other ones).

\section{Corollary 77 .}

(i) $\mathcal{C}_{\text {cont }}, \mathcal{C}_{\text {st }}$ and $\mathcal{G}_{\text {hycoh }}$ are pairwise incomparable.

(ii) $\mathcal{K}, \mathcal{C}_{s t}$ and $\mathcal{G}_{\text {hycoh }}$ are pairwise incomparable.

(iii) $\mathcal{K}, \mathcal{G}_{\text {coh }}$ and $\mathcal{G}_{\text {hycoh }}$ are pairwise incomparable.

(iv) $\mathcal{G}, \mathcal{G}_{\text {coh }}$ and $\mathcal{G}_{\text {hycoh }}$ are pairwise incomparable.

Thus, to answer positively the initial conjecture there only remains to prove:

\section{Conjecture 20 .}

(i) $\lambda \mathcal{C}_{\text {cont }}-\lambda \mathcal{C}_{\text {sts }} \neq \emptyset$; moreover it should be the case that:

(ii) $\operatorname{Th}(\mathcal{M}) \in \lambda \mathcal{C}_{\text {cont }}-\lambda \mathcal{C}_{\text {sts }}$, with $\mathcal{M}$ as in the proof of Theorem 76.

The reason why Bastonero could not conclude in his thesis that $T h(\mathcal{M}) \notin \lambda \mathcal{C}_{\text {sts }}$ is that he did not succeed to prove that a model of $\mathcal{C}_{\text {sts }}$ satisfying $F$ cannot satisfy $t_{1}=t_{2}$, with $t_{1}, t_{2}$ as in the proof of Theorem 76 . Indeed, in the case of $\mathcal{G}_{\text {hycoh }}$ (resp. $\mathcal{C}_{s t}$ ), he could exhibit a strongly stable function (resp. a stable function) $g$ separating $t_{1}$ and $t_{2}$ in all models of $F$; but his definition of $g$ relied, in the strongly stable case, on the fact that we were dealing with webbed models, which is not the case of all the models of $\mathcal{C}_{s t s}$ (while in the stable case the definition of $g$ was not problematic).

A wealth of incompleteness proofs for the three main semantics Salibra's Theorem 28 was a definite and uniform argument proving that each of main semantics is incomplete (and even $2^{\omega}$-incomplete). But, historically, the incompleteness of $\mathcal{C}_{\text {cont }}$ was first proved by Honsell and Ronchi, by producing an operational $\lambda$-theory which could not be the theory of a continuous model [26]. The proof, which was already quite technical, was adapted to the stable case by Gouy [23], but the new proof was so complicate that it became clear that one had to find a different idea for $\mathcal{C}_{\text {sts }}$. Such other approaches were provided by Bastonero and Gouy (cf. Theorems 75 and 76 above), which hence provided two new incompleteness proofs for each of $\mathcal{C}_{\text {cont }}, \mathcal{C}_{s t}$ and $\mathcal{G}_{h y c o h}$. To summarize, we have four different proofs for the incompleteness of $\mathcal{C}_{\text {cont }}$, also four for $\mathcal{C}_{s t}$, three for the subclass $\mathcal{G}_{\text {hycoh }}$ of $\mathcal{C}_{\text {sts }}$, and only Salibra's proof for $\mathcal{C}_{\text {sts }}$.

The last semantic problem. To have a more accurate picture of the relative position of $\lambda \mathcal{C}_{\text {cont }}, \lambda \mathcal{C}_{s t}$ and $\lambda \mathcal{C}_{\text {sts }}$, one should have an idea of the width and height of $\lambda \mathcal{T}-\lambda \mathcal{C}$, and of the width of $\lambda \mathcal{C}-\lambda \mathcal{C}^{\prime}$, for $\mathcal{C}, \mathcal{C}^{\prime}$ any distinct uniform 
classes of proper models. By Salibra's incompleteness theorem we know that for each ordered semantics we have that $\lambda \mathcal{T}-\lambda \mathcal{C}$ is $2^{\omega}$-high, but the question of the value of the height of $\lambda \mathcal{C}-\lambda \mathcal{C}^{\prime}$, for any other $\mathcal{C}^{\prime}$, is completely open, since we already know nothing about that of $\lambda \mathcal{C}$. On the contrary it is likely that the following conjecture (which only concerns uniform classes living in functional semantics) is true, and that all its instances will be accessible via generalized forcing.

Conjecture 21 For all $\mathcal{C}, \mathcal{C}^{\prime}$, either $\mathcal{C} \subseteq \mathcal{C}^{\prime}$ or $\lambda \mathcal{C}-\lambda \mathcal{C}^{\prime}$ is $2^{\omega}$-broad.

We can already give a positive answer to two instances of this conjecture. First we observe that generalized forcing is extendible to the extensional models of $\mathcal{K}$, in the same way usual forcing is (one just has to mix, in a straightforward way, the development of [30] or [5] with the one in [10]). Hence $\lambda \mathcal{K}^{e}$ is $2^{\omega}$-broad. Thus:

Proposition $78 \lambda \mathcal{K}-\lambda \mathcal{G}$ is $2^{\omega}$-broad.

Next we observe that, using Theorem 57 (generalized forcing), one can get for free $2^{\omega}$ versions of the model $\mathcal{M}$ of Theorem 76 in $\lambda \mathcal{G}-\left(\lambda \mathcal{C}_{s t} \cup \lambda \mathcal{G}_{\text {hycoh }}\right)$ with pairwise inconsistent theories $\left(\lambda \mathcal{K}^{e}\right.$ could also replace $\left.\lambda \mathcal{G}\right)$. Hence:

Proposition $79 \lambda \mathcal{G}-\left(\lambda \mathcal{C}_{s t} \cup \lambda \mathcal{G}_{\text {hycoh }}\right)$ is $2^{\omega}$-broad.

Proving the conjecture for other classes of the form $\lambda \mathcal{C}-\lambda \mathcal{C}^{\prime}$ supposes that we are able to prove by a forcing technique that this set is non empty, and that we can extend generalized forcing to $\lambda \mathcal{C}$.

\section{Conclusion.}

It is time to sum up the results which have been achieved these last four years.

\section{Answers to questions raised or quoted in [9, Section 6] .}

1. Salibra's Theorem 28 states that all the known ordered semantics are $2^{\omega}$-incomplete, which proves in particular that $\mathcal{C}_{\text {sts }}$ is incomplete. This answers very generously [Question 2 of Section $6.1, p .149$ ], which recalled the conjecture we had with Bastonero that $\mathcal{C}_{s t s}$ was incomplete (recall that Bastonero could only prove in his thesis that $\mathcal{G}_{\text {hycoh }}$ was incomplete). Salibra's proof was original, and initially inspired by universal algebra and general topology.

2. Bucciarelli and Salibra proved that $\lambda_{\beta}$ could not be the theory of a graph model, which answers the instance of [Question 1, Section 6.2, p.149] corresponding to $\mathcal{G}$.

3. Bucciarelli and Salibra's definition and study of the $\mathcal{G}$-product (cf. Section 5.3) allowed them to prove that there was a minimal graph-theory and a minimal sensible graph theory, and hence gave a positive answer to the instance of [Question 2 of Section 6.2, p.149] corresponding to $\mathcal{G}$ (and it also shows the 
way for larger classes of webbed models). But they leave open the question of whether the minimal sensible graph theory is $H$.

4. Bucciarelli and Salibra proved that the set of sensible graph theories has a greatest element, which furthermore is $B T$ (Theorem 63 ). This has the two following consequences. First, our [Question 1 of Section 6.3, p.151] can now be reformulated as follows: "Is $B T$ the only positive graph theory?". Second, combined with Kerth and David's theorem about the existence of $2^{\omega}$ sensible graph theories, it shows that there exists one, and even $2^{\omega}$, theory of sensible proper models below $B T$, which answers positively the two items of [Question 2 of Section 6.3, p.151]. Concerning the first question we conjecture in fact that the answer is positive, and proved some partial unpublished results with Salibra.

5. Starting from an example of Kerth (see Section 5.2), Alessi, Dezani and Honsell exhibited a result (the easiness of $\Omega_{3} I$ ), which admits a semantic proof using filter models, but for which there exists no semantic proof using $\mathcal{G}$ or $\mathcal{K}$ or, more generally, using any class of models whose underlying cpo is a prime algebraic domain. This also answers (part of) [Question 5 of Section 6.3, p.152] since it gives examples of theories represented in the continuous semantics, and via algebraic domains, but not via prime algebraic domains.

The four other recent results. The first to be mentioned was Statman's Theorem about the existence of a non trivial equation consistent with any consistent theory. The following two results answer questions raised by Antonino Salibra. In particular Salibra conjectured that the complete lattice $\lambda \mathcal{T}$ of all $\lambda$-theories does not satisfy any non trivial lattice identity, and proved that it is not modular, and hence not distributive. Then he proved with the author, using graph models, that there was a finitely axiomatizable $\lambda$-theory $T$ such that $[T, T o p]$ was a (very large) distributive lattice (see Section 5.3.6). For proving this, we generalized Baeten-Boerboom method of forcing, in a way which also

allowed us to prove that $\lambda \mathcal{G}$ was $2^{\omega}$-broad, as well as various smaller sets of graph theories. Also we are now in a position where we can conjecture that "all" the $\lambda \mathcal{C}^{\prime}-\lambda \mathcal{C}$ are $2^{\omega}$-broad, and prove some particular cases.

Partial results concerning the distributivity question had previously been obtained by Lusin and Salibra in [41] (existence of a lattice with weaker properties) and Alessi, Dezani and Lusin in [2] (existence of a filter model where $\Omega$ acts as union), with different methods.

Some long standing open questions still resist, like the representability of $H$, that of $\lambda_{\beta}$ and $\lambda_{\beta \eta}$ by reasonable proper models, and the question of the existence of a positive graph theory different from $B T$.

\section{References}

[1] F. Alessi, M. Dezani-Ciancaglini, F. Honsell, Filter models and easy terms, Italian Conference on Theoretical Computer Science, LNCS 
vol. 2202, Springer-Verlag, p.17-37, 2001.

[2] F. Alessi, M. Dezani-Ciancaglini, S. Lusin, Intersection types and domain operators, Theoretical Computer Science (to appear), 2004.

[3] J. Baeten and B. Boerboom, Omega can be anything it should not be, in Proceedings of the Koninklijke Nederlandse Akademie van Wetenschappen, Serie A, Indag. Mathematicae 41, p.111-120, 1979.

[4] H.P. Barendregt, The lambda-calculus, its syntax and semantics, Studies in Logic vol. 103, North-Holland, revised edition 1984.

[5] O. Bastonero, Modèles fortement stables du $\lambda$-calcul et résultats d'incomplétude, Thèse, Université de Paris 7, Dec. 1996.

[6] O. Bastonero, Equational incompleteness and incomparability results for $\lambda$-calculus semantics, 1998, unpublished but available at http://www.logique.jussieu.fr/www.bastoner.

[7] O. Bastonero and X. Gouy, Stabilité forte et incomplétude de la classe des modèles stables du $\lambda$-calcul, Notes de comptes-rendus de l'Académie des Sciences, t.322, n.10, série I, p.905-908, 1996.

[8] O. Bastonero and X. Gouy, Strong stability and the incompleteness of stable models of $\lambda$-calculus, Annals of Pure and Applied Logic, 100 (1999), pp. 247-277.

[9] C. Berline, From Computation to foundations via functions and application: The lambda-calculus and its webbed models, Theor. Comput. Sci. 249, p. 81-161, 2000.

[10] C. Berline and A. Salibra, Easiness in graph models, preprint, 34p. (2004), to appear in Theor. Comput. Sci.

[11] G. Berry, Stable models of typed lambda-calculi, In Proc. 5th Int. Coll. on Automata, Languages and Programming, LNCS 62, Springer-Verlag, Berlin (1978).

[12] A. Bucciareldi, Sequential models of PCF: some contributions to the domain-theoretic approach to full abstraction, Dottorato di ricerca in informatica, Università di Pisa-Genova-Udine, 1993.

[13] A. Bucciarelli and T. Ehrhard, Sequentiality and strong stability, Sixth Annual IEEE Symposium on Logic in Computer Science (1991), pp. 138-145.

[14] A. Bucciarelli and A. Salibra, The minimal graph-model of lambdacalculus, 28th Internat. Symp. on Math. Foundations of Comput. Science, LNCS 2747, Springer-Verlag, 2003. 
[15] A. Bucciarelli and A. Salibra, The sensible graph theories of lambdacalculus, 19th Annual IEEE Symposium on Logic in Computer Science (LICS'04), Turku, Finland, 2004.

[16] M. Coppo, M. Dezani-Ciancaglini, F. Honsell and G. Longo, Extended type structures and filter lambda models, Logic colloquium '82 (Florence, 1982), North-Holland, Amsterdam (1984) 241-262.

[17] R. DAvid, Every unsolvable $\lambda$-term admits a decoration, Proc. TLCA'99, Lecture Notes in Computer Science, vol. 1581, Spreinger, Berlin, 1999.

[18] R. DAvid, Computing with Böhm trees, Fundamenta Informaticae 45 p. 53-77, 2001.

[19] G. Durfy, A Model for a list-oriented Extension of the Lambda Calculus, Master Thesis, Carnegie Mellon 1997.

[20] G. Franco, Some intentional models of lambda-calculus, Tesi di Dottorato di Ricerca, Università degli Studi di Udine, Dipartimento di Matematica e Informatica, 2001.

[21] P. Di Gianantonio, F. Honsell, G. Plotkin, Uncountable limits and thye $\lambda$-calculus, Nordic. J. Comput. 2 (1995), 126-145.

[22] E. Engeler, Algebras and combinators, Alg. Univ. 13 (3), p.289-371, 1981.

[23] X. Gouy, Etude des théories équationnelles et des propriétés algébriques des modèles stables du $\lambda$-calcul, Thèse, Université de Paris 7, Dec. 1995.

[24] X. Gouy, Une extensiondu Théorème de Hyland et Wadsworth à une classe de modèles du $\lambda$-calcul pur, Notes C.R.Acad. Sci.Sér. I, 322, p. 419-422, 1996.

[25] F. Honsell and D. Sanella, Pre-logical relations, Proc. Computer Science Logic, CSL'99, Madrid, Springer LNCS 1683, 546-561 (1999).

[26] F. Honsell and S. Ronchi della Rocca, An Approximation Theorem for Topological Incompleteness of Lambda Calculus, Journal of Computer and System Sciences 45, p. 49-75, 1992.

[27] R. Hyland, A syntactic characterization of the equality in some models of the $\lambda$-calculus, J. London Math. Soc. 12, p. 361-370, 1976.

[28] G. Jacopini and M. Venturi Zilli, Easy terms in the $\lambda$-calculus, Fund. Informartica 80, 225-233, 1985.

[29] Y. JiAng, Consistance et inconsistance de théories de $\lambda$-calcul étendus, Thèse, Université Paris 7, Juin 1993. 
[30] Y. JiAng, Consistency of a $\lambda$-theory with n-tuples and easy terms, Archives of Math. Logic, vol.34 n.2, p.79-96, 1995.

[31] T.Jim, A.Meyer: Full abstraction and the context lemma, in TACS 1991, Sendai, LNCS 526, Spinger, 1991.

[32] R. KeRTH, $2^{\aleph_{0}}$ modèles de graphes non équationnellement équivalents, Notes de comptes-rendus de l'Académie des Sciences 318, p.587-590, 1994.

[33] R. KERTH, Isomorphisme et équivalence équationnelle entre modèles du $\lambda$-calcul, Thèse, Université de Paris 7, Oct. 1995.

[34] R. KERTH, The interpretation of unsolvable terms in models of pure $\lambda$ calculus, J. of Symbolic Logic.63, p.1529-1548, 1998.

[35] R. KeRTh, Isomorphism and equational equivalence of continuous $\lambda$ models, Studia Logica 61, 403-415, 1998.

[36] R. KERTh, Forcing in stable models of untyped $\lambda$-calculus, Indagationas Mathematicae 10 , p.59-71, 1999.

[37] R. KerTh, On the construction of stable models of untyped $\lambda$-calculus, Theor. Comput. Sci. 269, 1-2, p.23-46, 2001.

[38] J.L. KRivine, $\lambda$-calcul, types et modèles, Masson, Paris, 1990.

[39] J.L. Krivine, $\lambda$-calcul, types and models, Ellis-Horwood, Chichester, 1993 (augmented english translation of the above).

[40] G. Longo, Set-theoretical models of $\lambda$-calculus : theories, expansions and isomorphisms, Annals of Pure and Applied Logic 24, p.153-188, 1983.

[41] S. Lusin And A. Salibra, The lattice of Lambda Theories, J. of Logic and Computation, Vol.14 n ${ }^{\circ}$, p.373-394, 2004.

[42] R.N. McKenzie, G.F. McNulty, W.F. Taylor, Algebras, Latticies, Varieties, Vol I, Wadsworth \& Brooks, Monterey, California,1987.

[43] G.D Plotkin, A set-theoretical definition of application, Memorandum MIP-R-95, School of artificial intelligence, University of Edinburgh, 1972.

[44] G.D Plotkin, LCF as a programming language, Theoret. Comput. Sci 5 (1977), 223-257.

[45] G.D. Plotkin, Set-theoretical and other elementary models of the $\lambda$ calculus, Theoretical Computer Science 121 (1993) p.351-409.

[46] G.D. Plotkin, On a question of H. Friedman, Information and Computation, 126(1), p.74-77, 1996.

[47] A. Salibra, On the algebraic models of $\lambda$-calculus, Theoretical Computer Science 249, p.197-240, 2000. 
[48] A. Salibra, Nonmodularity results for lambda-calculus, Fundamenta Informaticae 45, p.379-392, 2001.

[49] A. SAlibra, A continuum of theories of lambda calculus without semantics, 16th Annual IEEE Symposium on Logic in Computer Science, Boston, USA, 2001.

[50] A. SAlibra, Topological incompleteness and order incompleteness of the lambda calculus, ACM Transactions on Computational Logic 4, p.379-401, 2003.

[51] D.S. Scott, Continuous lattices, Toposes, Algebraic geometry and Logic, Lecture Notes in Mathematics 274, Springer-Verlag, Berlin, 1972.

[52] P. Selinger, Order-incompleteness and finite $\lambda$-models, in Proc. 11th Annual IEEE Symp. on Logic and Computer Science, LICS'96 (1996), p. 422-439.

[53] P. Selinger, Order-incompleteness and finite lambda reduction models, Theoretical Computer Science 309 p. 43-63, 2003.

[54] R. Statman, Marginalia to a Theorem of Jacopini, Fundamentae Informaticae 2001, vol. 45, issue 1,2, p.117-121.

[55] A. Visser, Numerations, $\lambda$-calculus and arithmetic, To H.B. Curry: Essays on Combinatory Logic, Lambda-Calculus and Formalism (J.R. Hindley and J.P. Seldin eds.), Academic Press, New York, p. 259-284, 1980.

[56] C. Wadsworth, The relation between computational and denotational properties for $D_{\infty}$-models of the lambda-calculus, SIAM Journal of Computing 5 p. $488-521,1976$.

[57] C. Zylberajch, Syntaxe et sémantique de la facilité en $\lambda$-calcul, Thèse, Université Paris 7, 1991. 